HD-THEP-96-31

HD-TVP-96-8

\title{
Vector meson leptoproduction and nonperturbative gluon fluctuations in QCD
}

\author{
H.G. Dosch, T. Gousset*, G. Kulzinger ${ }^{\dagger}$, H.J. Pirner \\ Institut für Theoretische Physik der Universität Heidelberg, \\ Philosophenweg 16 \&3 19, D69120 Heidelberg
}

\begin{abstract}
We present a nonperturbative QCD calculation of diffractive vector meson production in virtual photon nucleon scattering at high energy. We use the nonperturbative model of the stochastic QCD vacuum which yields linear confinement and makes specific predictions for the dependence of high-energy scattering cross sections on the hadron size. Using light cone wave functions of the photon and vector mesons, we calculate electroproduction cross sections for $\rho, \omega, \phi$ and $J / \psi$. We emphasize the behavior of specific observables such as the ratio of longitudinal to transverse production cross section and the $t$-dependence of the differential cross section.
\end{abstract}

*Supported by the Federal Ministry of Education, Science, Research and Technology (BMBF) under grant no. 06 HD 742

${ }^{\dagger}$ Supported by the Deutsche Forschungsgemeinschaft under grant no. GRK 216/1-96 


\section{INTRODUCTION}

Exclusive vector meson production by real and virtual photons is a good probe to investigate the physics of diffractive scattering. Whenever the coherence length of the photon is larger than the proton radius, it is preferable to study the process in the proton rest frame or in the center of mass frame where the virtual photon can be considered as a hadronic system composed of partons. In this case the photon-hadron interaction is then in many respects similar to hadron-hadron collision. In addition, it offers the possibility to vary the polarization and virtuality of the photon and thereby manipulate the light cone wave function of the incoming state. "The experimentalist can make hadrons of arbitrary sizes."

In our approach, we attack the problem as a genuine nonpertubative one. We use the model of the stochastic vacuum [1] which has been adapted to high-energy hadron-hadron scattering in Ref. [2], applying the general scheme developped by Nachtmann [3] for the separation of the large energy scale from the small scale of momentum transfer. The model of the stochastic vacuum gives satisfactory results both in low and high energy physics. It yields a rather simple geometrical picture for a single gluonic flux tube $\mathbb{1}$. The same mechanism of nonperturbative gluon fluctuations which leads to confinement also generates an interaction of the strings in colliding hadrons. In this picture hadron-hadron scattering cannot be constructed from quark-quark scattering since the string-string interaction plays an important role. It leads to cross sections which are determined by the transverse extensions of the interacting hadrons. In addition to the forward scattering amplitudes, the model provides the $t$-dependence of the cross section explaining the phenomenologically observed [5] correlation between elastic slopes and total cross sections.

Soft electroproduction on the nucleon can be calculated along the same lines as hadronnucleon scattering using a model wave function for the photon. At small $Q^{2}$, the photon is of hadronic size and large-distance-physics as in hadron-hadron scattering should apply. In another paper [6], we construct the wave function of the photon as a superposition of vector meson states and calculate the production cross section of $\rho, \rho^{\prime}$ and $\rho^{\prime \prime}$. This approach is limited to virtualities $Q^{2} \lesssim 2 \mathrm{GeV}^{2}$ and the unknown couplings of the electromagnetic current to the $\rho^{\prime}$ and $\rho^{\prime \prime}$ introduce new parameters. In this paper, we explore the possibility to represent the incoming photon as a $q \bar{q}$ state. With increasing $Q^{2}$, the transverse extension of the $q \bar{q}$ dipole diminishes in a way that depends on the polarization of the virtual photon. We shall demonstrate how this mechanism shows up phenomenologically. This allows us to study the transition from large to short-distance-dominated-processes. The cross section for transversely polarized photons has a large non perturbative part, because endpoints at momentum fraction $z=0$ and $z=1$ in the photon wave function do not select a $q \bar{q}$ system of small transverse separation. Therefore our large distance mechanism is important here, too.

In our model the length of the string connecting the valence quarks in the hadron turns out to be very important. This length depends on the light cone wave function of the hadron. There is yet little knowledge about the physics determining the light cone Hamiltonian in nonperturbative QCD. So at the moment these wave functions and their integrated distribution amplitudes fulfill mainly a phenomenological task to parametrize the valence quark content of the hadron. Although, the exact value of cross section depends in our model on the detailed form of the wave function, the $Q^{2}$-behavior of specific observables such as ratios 
of longitudinal to transverse vector meson production or elastic slopes are likely to provide a good test for the string picture inherent in our model.

Electroproduction of vector mesons has also been discussed within a soft pomeron framework in Ref. [7,8]. In this model, transverse sizes of hadrons only play a marginal role because, on the one hand, hadron scattering can systematically be reduced to quark scattering through the property of quark additivity of the model and, on the other hand, the vector meson wave functions are assumed to be wider than the distance of the quarks in the virtual photon and are thus replaced by their value at the origin. These assumptions are phenomenologically tenable if one further assumes that the quark-pomeron coupling is flavor dependent. At intermediate $Q^{2}=1-10 \mathrm{GeV}^{2}$ [7], a pomeron form factor is used for far-off-shell quark legs. At larger values of $Q^{2}$ [8], nonperturbative two-gluon exchange is applied which leads to color singlet cancellation at small $q \bar{q}$-dipole size.

In a series of papers [9], a perturbative two-gluon exchange model extended to include nonperturbative effects via the gluon distribution in the proton has been developped to evaluate vector meson production. This approach has been further refined to incorporate a BFKL-like evolution to accomodate both energy and $Q^{2}$-dependence. Dipole scattering is the basis of this framework. In the following paper we are treating the photon and vector meson in a similar way. The main difference between our approaches lies in the reaction mechanism for soft diffraction.

The importance of the gluon distribution as a necessary part of hard diffraction has been advocated in Ref. [10,11]. These authors have limited the range of applicability of their perturbative calculations to reactions where a large transverse momentum scale rules the exchanges, i.e. to heavy quark production such as $J / \psi$-production $\left[10 \|\right.$ or at large $Q^{2}$ $\left(Q^{2} \geq 10 \mathrm{GeV}^{2}[11)\right.$.

HERA has opened up new possibilities to enlarge the energy and $Q^{2}$ range. Recent ZEUS and $\mathrm{H} 1$ data indicate that at large $Q^{2}$ the cross section may rise more steeply than expected from soft pomeron exchange. A possible explanation in the language used above [12] is that the evolution of the wavefunction at higher energy and $Q^{2}$ gives rise to more and more dipoles inside the hadron. This evolution would then be a prerequisite to discuss the energy dependence of the virtual photon cross section with increasing resolution. This phenomenon of "hard" pomeron exchange will not be addressed in the following study which deals with soft pomeron physics at a fixed energy.

The kinematics are defined in Fig. 1. We denote the initial photon 4-momentum with $q$, the initial nucleon momentum with $p$, and the equivalent final states with $q^{\prime}$ and $p^{\prime}$. $\Delta=q^{\prime}-q$ is the momentum transfer and the independent Lorentz invariants are

$$
\begin{aligned}
s & =(p+q)^{2}, \\
t & =\Delta^{2}=\left(q^{\prime}-q\right)^{2}, \\
Q^{2} & =-q^{2} .
\end{aligned}
$$

We are interested in soft reactions, i.e. $|t|<1 \mathrm{GeV}^{2}$, at high energy, $s \gg Q^{2}$ and $s \gg M_{p}^{2}$ (e.g. $s>100 \mathrm{GeV}^{2}$ ). In this domain, $x_{\mathrm{B}}=Q^{2} / 2 p . q$ is small, e.g. $x_{\mathrm{B}}<0.1$.

To be specific, we use the center-of-mass frame where the photon momentum points along the $z$-axis. Then the absolute sizes of the 3 -momenta are given as 


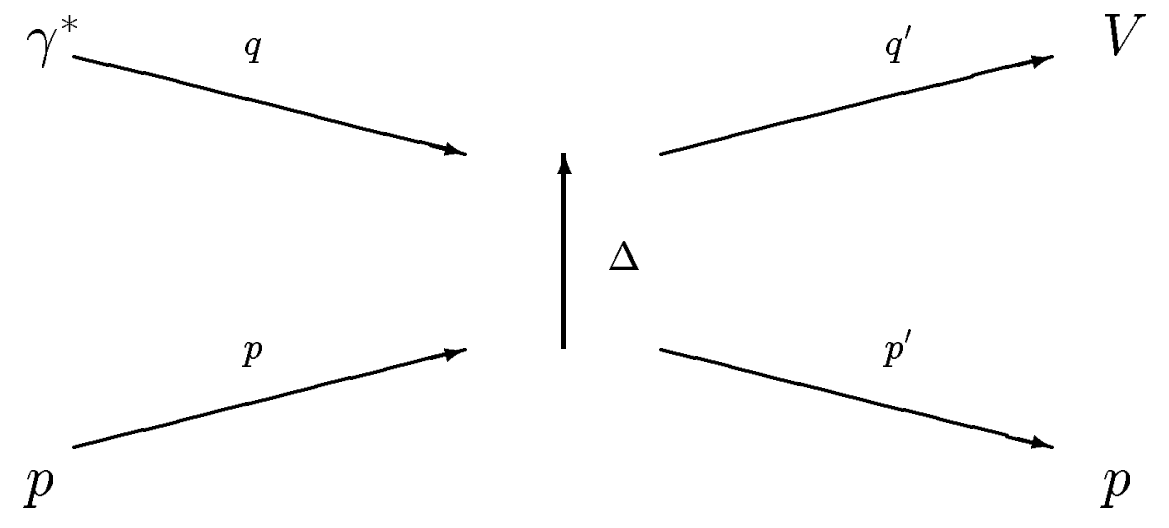

FIG. 1. Kinematics of the reaction $\gamma^{*}+p \rightarrow V+p$.

$$
\begin{gathered}
|p|=|q|=\frac{\sqrt{s}}{2}+\frac{Q^{2}-M_{p}^{2}}{2 \sqrt{s}}+O\left(s^{-3 / 2}\right), \\
\left|p^{\prime}\right|=\left|q^{\prime}\right|=\frac{\sqrt{s}}{2}-\frac{M_{V}^{2}+M_{p}^{2}}{2 \sqrt{s}}+O\left(s^{-3 / 2}\right) .
\end{gathered}
$$

In this frame the vector meson emerges with a small transverse momentum $\left|\mathbf{q}_{T}^{\prime}\right|=\Delta_{T} \approx$ $\theta \sqrt{s} / 2<1 \mathrm{GeV}$. Unlike in the elastic scattering case, the center of mass momentum varies in this reaction

$$
\delta=|p|-\left|p^{\prime}\right| \approx \frac{Q^{2}+M_{V}^{2}}{2 \sqrt{s}} .
$$

This implies that the momentum transfer $\Delta$ has a time component $\Delta^{0} \approx \delta$, besides the space components $\Delta^{z} \approx-\delta-\sqrt{s} \theta^{2} / 4$ and $\Delta_{T} \approx \sqrt{s} \theta / 2$. Let us notice for completness that the square of $\Delta$ is $t=\Delta^{2} \approx-\Delta_{T}^{2}+t_{0}$ with $t_{0}=-M_{p}^{2}\left(Q^{2}+M_{V}^{2}\right)^{2} / s^{2}$. In the high energy limit $s \gg Q^{2}+M_{V}^{2}$ the space components dominate. When we demand in addition a finite transfer, i.e. a small scattering angle $\theta / 2=O(1 / \sqrt{s})$, the transverse component is the leading component of $\Delta$. In the following we shall therefore neglect all component besides $\boldsymbol{\Delta}_{T}$.

The outline of the paper is as follows. Section II gives a very short and non-technical description of the model of the stochastic vacuum and describes its application to high-energy scattering. Section III deals with the specific features of electroproduction, i.e. the photon and vector meson wave functions, which are used in the evaluation of the longitudinal and transverse cross sections. Sections IV contains the numerical results for $\rho, \phi, J / \psi$ integrated and differential cross sections as functions of $Q^{2}$. As far as possible these results are compared to experiment. Section V concludes with a discussion of the results.

\section{HIGH-ENERGY ELASTIC SCATTERING IN THE STOCHASTIC VACUUM}

\section{A. The model of the stochastic vacuum}

The model of the stochastic vacuum is based on the asumption that the contributions of the slowly varying gluon fields in an infrared regular QCD can be approximated by a simple 
stochastic process (for a review see Ref. [13]). Already the assumption that this process has a converging cluster expansion leads to linear confinement in a non-Abelian gauge theory. As usual, approximations to a quantum field theory in the functional approach are more safely made in an Euclidean rather than Minkowskian field theory. It turns out however that in high-energy scattering there is no feasible way to continue Greens functions from the Euclidean to the Minkowskian world and we therefore have to formulate the model in the Minkowski continuum. This seems at first sight more dramatic than it turns out to be finally, since at the end we have to evaluate the relevant quantities only at spacelike Euclidean distances, i.e we can take these quantities from an Euclidean field theory.

In order to define gauge invariant correlators we introduce the modified gluon field strength $F_{\mu \nu}(x, \omega)$ which is obtained from the field strength at point $x$ by parallel transporting the colour content to the point $\omega$

$$
F_{\mu \nu}(x, \omega)=\Phi^{-1}(x, \omega) F_{\mu \nu}(x) \Phi(x, \omega),
$$

with $\Phi(x, \omega)=P \exp \left[-i g \int_{\omega}^{x} A d z\right]$.

Assuming that the main features of the correlator $\left\langle F_{\mu \nu}(x, \omega) F_{\rho \sigma}(y, \omega)\right\rangle$ do not depend crucially on the choice of the reference point $\omega$ we obtain for the dependence on $z=x-y$ the most general form

$$
\begin{aligned}
\left\langle g^{2} F_{\mu \nu}^{c}(x, \omega) F_{\rho \sigma}^{d}(y, \omega)\right\rangle_{A}= & \frac{\delta^{c d}}{N_{c}^{2}-1} \frac{1}{12}\left\langle g^{2} F F\right\rangle\left\{\kappa\left(\eta_{\mu \rho} \eta_{\nu \sigma}-\eta_{\mu \sigma} \eta_{\nu \rho}\right) D\left(z^{2} / a^{2}\right)\right. \\
& \left.+(1-\kappa) \frac{1}{2}\left[\partial_{\mu}\left(z_{\rho} \eta_{\nu \sigma}-z_{\sigma} \eta_{\nu \rho}\right)+\partial_{\nu}\left(z_{\sigma} \eta_{\mu \rho}-z_{\rho} \eta_{\mu \sigma}\right)\right] D_{1}\left(z^{2} / a^{2}\right)\right\} .
\end{aligned}
$$

The correlator $D$ is typical for a non-Abelian gauge theory (or an Abelian theory with monopoles) since the homogeneous Maxwell equations

$$
\epsilon^{\mu \nu \rho \sigma} \partial_{\nu} F_{\rho \sigma}=0
$$

allow only the tensor structure proportinal to $D_{1}$, hence $\kappa=0$ in an Abelian theory without monopoles.

In a Gaussian model, where all higher cumulants in the linked cluster expansion [14] are neglected, we obtain a relation between the slope of the static quark-antiquark potential and the typically non-Abelian correlator $D$

$$
\sigma=\kappa \frac{\pi}{144}\left\langle g^{2} F F\right\rangle a^{2} \int_{0}^{+\infty} d u D(-u)
$$

The choice of phenomenological parameters will be given in Sec. II .

\section{B. Scattering of two color singlet dipoles}

The high-energy scattering of two color singlet dipoles $q_{1} \bar{q}_{1}$ and $q_{2} \bar{q}_{2}$ can be treated analogously to the situation of heavy quarks encountered in the Wilson area law. The relativistic quarks and antiquarks move along two opposite straight line trajectories on the light cone. 


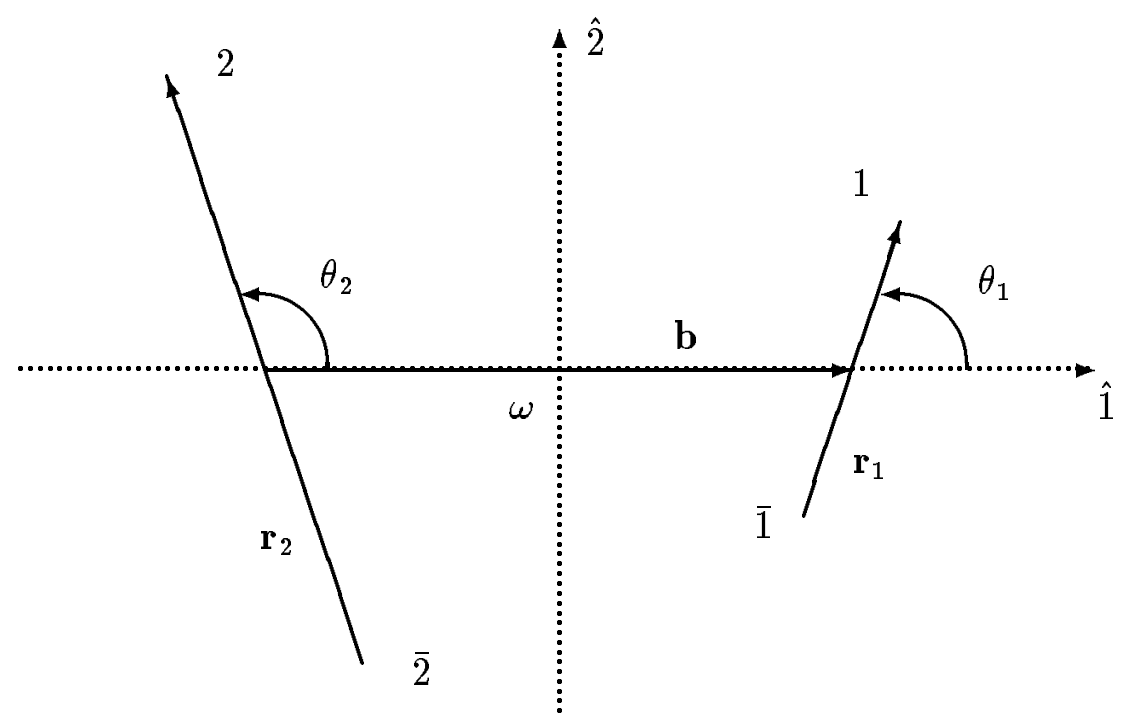

FIG. 2. Configuration of the two interacting loops in the transverse plane. With our choice of frame, the loops 1 and 2 lie, in the $\left(x^{0}, x^{3}\right)$-plane, on the lines $x^{0}=x^{3}$ and $x^{0}=-x^{3}$ respectively.

In order to apply the model of the stochastic vacuum to high-energy hadron hadron scattering we adopt the method of Ref. [3]. In this approach the problem is first considered as the scattering of quarks in an external colour field which is solved for fast moving quarks by the leading term of an eikonal expansion, i.e. the quark picks up the eikonal phase

$$
V=\exp \left[-i g \int_{\Gamma} A d z\right]
$$

where $\Gamma$ is the classical path of the quark.

This phase is manifestly gauge dependent, but if we consider a fast moving dipole, i.e. a quark and an antiquark moving on parallel lightlike trajectories connected by a Schwinger string, then we have to evaluate rather a Wilson loop

$$
W=\exp \left[-i g \int_{\partial S} A d z\right]
$$

than the path integral above. The open ends of the $q$ and $\bar{q}$ trajectories in dipole 1 and dipole 2 are closed by small transverse lines yielding two loops, $\partial S_{1}$ and $\partial S_{2}$, which have transverse extensions according to the lengths of the dipoles $r_{1}$ and $r_{2}$. The dipoles are positioned relative to each other with a given impact parameter $b$. The loop-loop interaction amplitude for this system of dipoles is calculated in Ref. [2]

$$
J\left(\mathbf{x}_{1}, \mathbf{x}_{\overline{1}}, \mathbf{x}_{2}, \mathbf{x}_{\overline{2}}\right)=\left\langle\frac{1}{N_{C}} \operatorname{tr}\left(W_{1}\left(\mathbf{x}_{1}, \mathbf{x}_{\overline{1}}\right)-1\right) \frac{1}{N_{C}} \operatorname{tr}\left(W_{2}\left(\mathbf{x}_{2}, \mathbf{x}_{\overline{2}}\right)-1\right)\right\rangle_{A},
$$

where the bold faced vectors $\mathbf{x}_{i}=\left(x_{i}^{1}, x_{i}^{2}\right)$ denote the two-dimensional positions of quarks $i=1,2$ in the transverse plane ( $\bar{\imath}$ refers to the corresponding antiquarks). The geometry of the loops is shown in Fig. 2.

The various steps and approximations necessary to derive a tractable expression of $J$ have been developped in Ref. [2] to which we direct the interested reader. Here we only 
summarize these steps. First one transforms the line integrals appearing in the non abelian phases $W_{1}$ and $W_{2}$ into surface integrals. The manipulation of gauge invariant quantities leads to the introduction of a reference point $\omega$ in between the two surfaces. The surfaces to be considered are two pyramids, $S_{1}$ and $S_{2}$, with $\omega$ as apex and $\partial S_{1}, \partial S_{2}$ as respective basis. This is shown in Fig. 3. The surfaces are the world sheets of infinitely many gluons in the two-hadron state which interact via the correlator Eq. (2.1). In order to make the calculation practical, these interactions are truncated to fourth order in the field strengths. Because of the Gaussian process the terms factorize into products of integrals over the correlators linking the surfaces generated by $W_{1}$ and $W_{2}$. One gets

$$
J \approx \frac{1}{8 N_{C}^{2}\left(N_{C}^{2}-1\right)}\left(\int_{S_{1}} d \Sigma^{\mu \nu}(x) \int_{S_{2}} d \Sigma^{\rho \sigma}(y)\left\langle g^{2} F_{\mu \nu}^{c}(x, \omega) F_{\rho \sigma}^{c}(y, \omega)\right\rangle_{A}\right)^{2} .
$$

Symbolically, this expression can be rearranged into a sum of four interaction-terms, $\chi$,

$$
J=\frac{1}{8 N_{C}^{2}\left(N_{C}^{2}-1\right)}\left(\frac{\left\langle g^{2} F F\right\rangle}{12}\right)^{2}\left\{\chi\left(q_{1} q_{2}\right)+\chi\left(\bar{q}_{1} \bar{q}_{2}\right)-\chi\left(q_{1} \bar{q}_{2}\right)-\chi\left(\bar{q}_{1} q_{2}\right)\right\}^{2},
$$

where each term has confining and non-confining parts from the basic gluon-gluon correlator in the vacuum, $\chi(q q)=\kappa \chi_{c}(q q)+(1-\kappa) \chi_{n c}(q q)$, which we now specify.

It is possible to choose functions $D$ and $D_{1}$ in a way that the natural fall of $\mathrm{F}$ at large Euclidean distances does not impede a meaningful continuation to Minkowski space. A possible choice consistent with confinement and lattice computation [15] is

$$
D\left(z^{2} / a^{2}\right)=\frac{27 \pi^{4}}{4} i \int \frac{d^{4} k}{2 \pi^{4}} \frac{k^{2}}{\left(k^{2}-(3 \pi / 8)^{2}\right)^{4}} e^{-i k \cdot z / a} .
$$

The function $D_{1}$ is a priori an independent one, however lattice simulation indicates that the behavior of $D$ and $D_{1}$ is similar and we shall assume for simplicity that $D_{1}=D$.

For the non-confining part the two surface integrals can be performed and result in quark-quark interaction terms such as

$$
\chi_{n c}\left(q_{1} q_{2}\right)=\frac{8}{3}\left(\frac{\left|\mathbf{x}_{1}-\mathbf{x}_{2}\right|}{a}\right)^{3} K_{3}\left(\frac{3 \pi}{8} \frac{\left|\mathbf{x}_{1}-\mathbf{x}_{2}\right|}{a}\right) .
$$

Since nonperturbative gluon correlations are of size $a$, two color charges can only interact if their trajectories enter in a common domain of size $a$. A constituent quark picture arises where the elementary color charges are surrounded with gluon clouds. This can be seen in Fig. $1(\mathrm{a})$ where the interaction amplitude $J$ for $\kappa=0$ between a dipole target of size $r_{2}=12 a$ oriented along a given $x$-axis and a dipole probe of size $r_{1}=a$ is plotted as a function of the impact position. For simplicity we sum over the orientation of the probe. It turns out that with a physical correlation length around $0.3 \mathrm{fm}$ a physical target has a size of $4 a-5 a$ so that constituents in the target are not as well separated as in Fig. 4 .

For the confining part on the contrary the integrals have a path dependence which is linked to the non-abelian nature of the confining term. Physically this means that the color dipoles connected by their strings interact as whole objects rather than as isolated endpoints. In space-time the integration over the surfaces is done with the reference point $\omega$ chosen in 

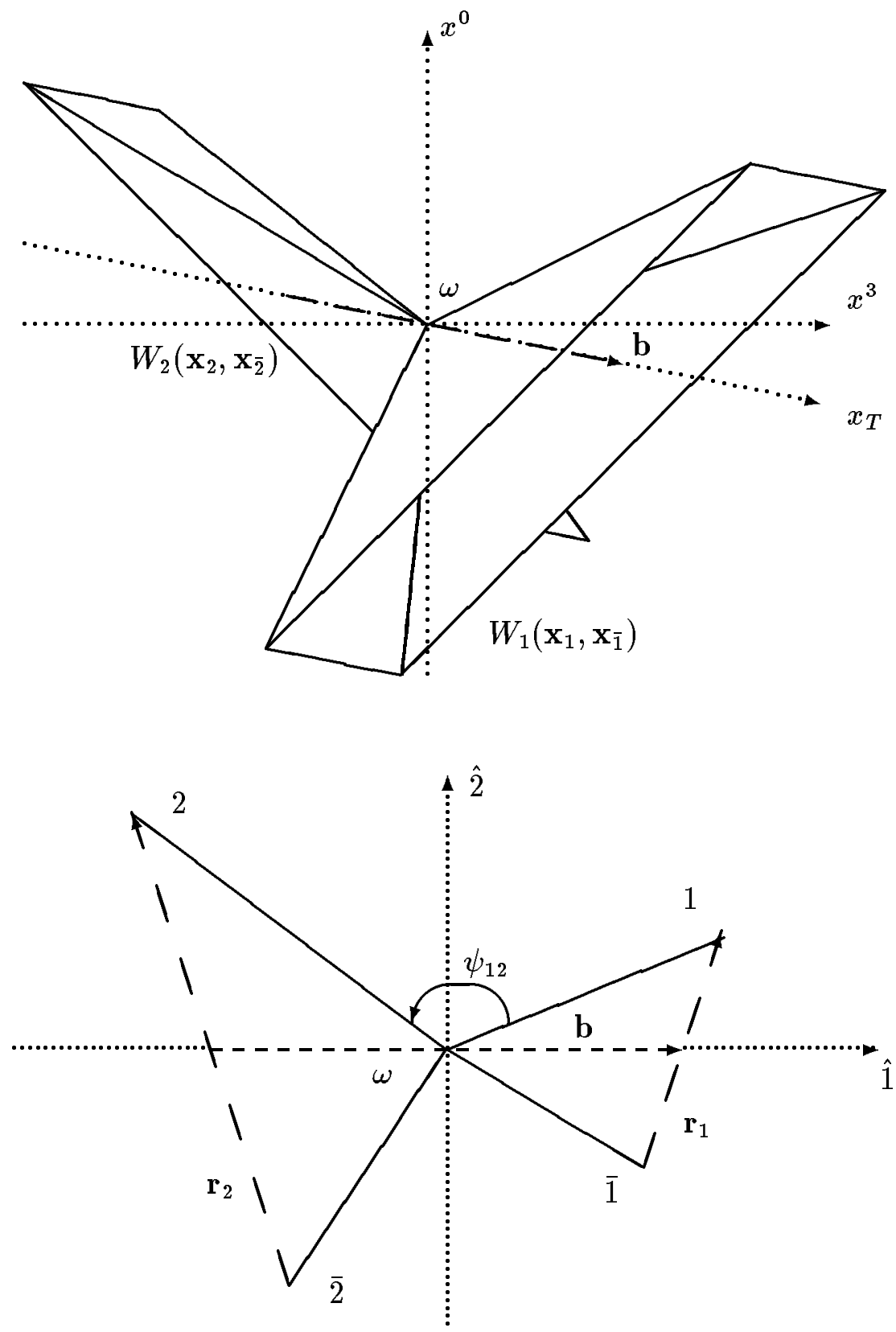

FIG. 3. Space-time representation of the pyramids and their transverse projection. The sliding sides of the pyramids give the domains $S_{1}$ and $S_{2}$ of the surface integrations. Note that the two Wilson loops are not parallel in the transverse plane. 

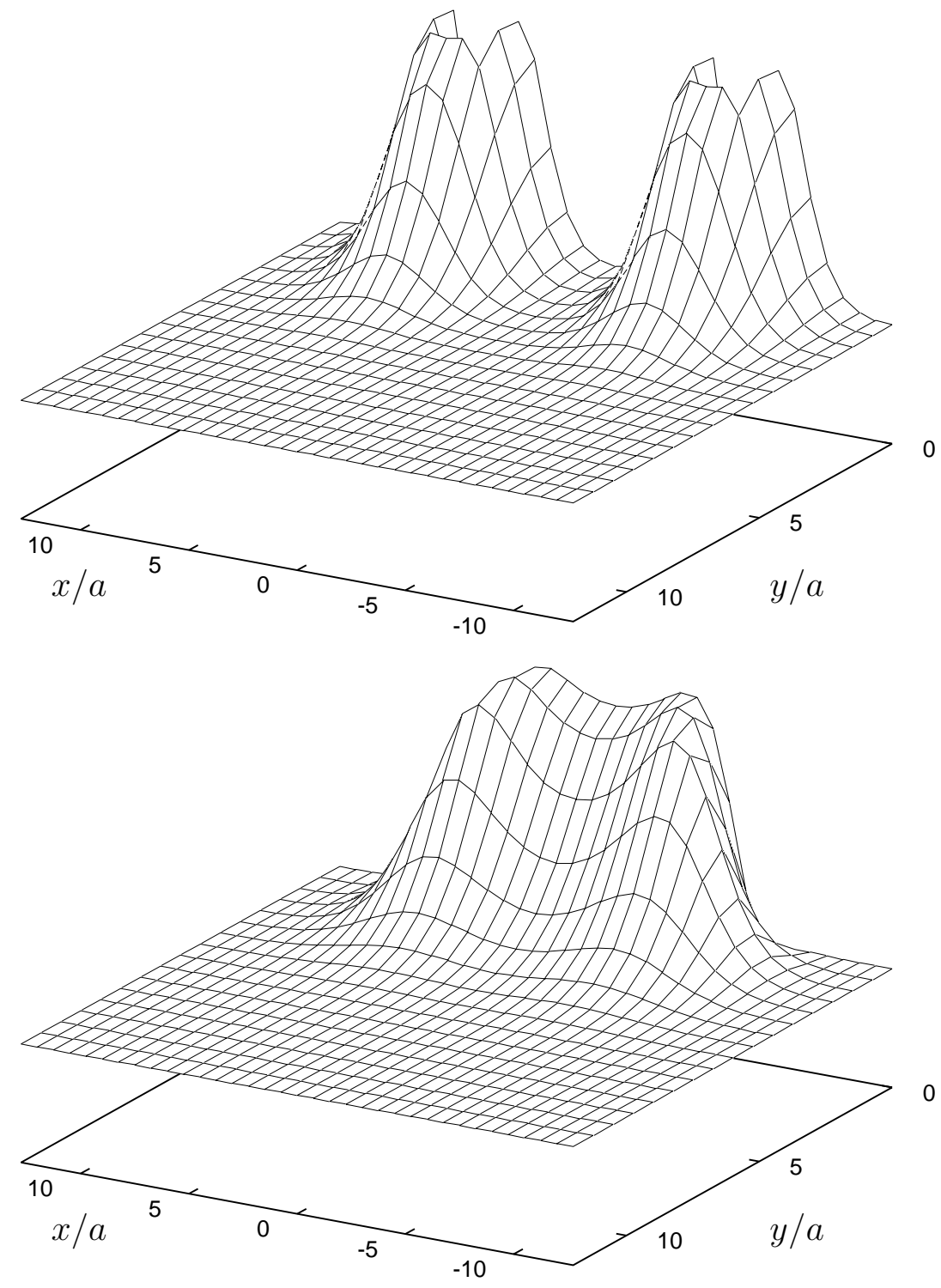

FIG. 4. (a) Color interaction amplitude Eq. (2.2) for the non-confining case, $\kappa=0$, as a function of the impact position between the two dipoles. Dipole 1 has a transverse size $r_{1}=a$ and we sum over its orientation. Dipole 2 has a transverse size $r_{2}=12 a$ and lies along the $x$-axis. (b) Color interaction amplitude for the confining case, $\kappa=1$. 
the most symmetrical way as shown in Fig. 3. The result depends only very weakly on the choice of the reference point and has the following form:

$$
\begin{aligned}
& \chi_{c}\left(q_{1} q_{2}\right)=\frac{\pi}{2} \cos \psi_{12} \\
& \left\{\frac{\left|\mathbf{x}_{1}-\mathbf{x}_{\omega}\right|}{a} \int_{0}^{1} d \alpha\left|\frac{\mathbf{x}_{1}-\mathbf{x}_{\omega}-\alpha\left(\mathbf{x}_{2}-\mathbf{x}_{\omega}\right)}{a}\right|^{2} K_{2}\left(\frac{3 \pi}{8 a}\left|\mathbf{x}_{1}-\mathbf{x}_{\omega}-\alpha\left(\mathbf{x}_{2}-\mathbf{x}_{\omega}\right)\right|\right)+(1 \leftrightarrow 2)\right\} .
\end{aligned}
$$

The angle $\psi_{12}$ denotes the angle between the vectors $\mathbf{x}_{1}-\mathbf{x}_{\omega}$ and $\mathbf{x}_{2}-\mathbf{x}_{\omega}$. The amplitude in the string-string interaction picture, i.e. $\kappa=1$, is shown in Fig. 4 (b) with the same choice for the target and probe sizes as in the non-confining case. The interaction is non-zero whenever the probe is close to the line connecting the target quark and antiquark. We note that the string and constituent picture both differ from the optical droplet picture where the charge distribution form factor is responsible for the differential cross section. Via the wave functions of the valence quarks the geometrical sizes of the hadrons enter in the cross sections.

\section{From dipole-dipole to hadron-hadron cross section}

A valence quark picture can be constructed from nonperturbative scattering amplitude of color dipoles with fixed lengths $r_{1}$ and $r_{2}$ by distributing the positions of the end-points of the strings according to a quantum mechanical wave function. Since for high-energy scattering the incoming particles propagate along the light cone, it is natural to choose light cone wave functions. The amplitude of the process can be written as 16

$$
\mathcal{M}=2 i s \int d^{2} \mathbf{b} e^{-i \boldsymbol{\Delta}_{T} \cdot \mathbf{b}} \int \frac{d z_{1} d^{2} \mathbf{r}_{1}}{4 \pi} \psi_{V}^{\dagger} \psi_{\gamma}\left(z_{1}, \mathbf{r}_{1}\right) \int \frac{d z_{2} d^{2} \mathbf{r}_{2}}{4 \pi}\left|\psi_{p}\left(z_{2}, \mathbf{r}_{2}\right)\right|^{2} J\left(\left\{\mathbf{x}_{i}\right\}\right),
$$

where the index " 1 " refers to the photon or vector meson side whereas the index " 2 " is attached to the nucleon coordinates. (Conventions are fixed in Appendix A.) For simplicity, the nucleon is considered in a quark-diquark configuration. It has been shown in Ref. [2] that quark-diquark and three-quark pictures lead to similar predictions for diffractive scattering once the model parameters are adjusted to fit the proton-proton cross section. In the following, we also fix the parameters to fit proton-proton cross section and therefore we do not expect any significative dependence on the model of the proton. For $C=P=-1$ exchange, the quark-diquark is favoured since it suppresses the odderon contribution [17].

The $\psi$ 's are the valence light cone wave functions of the corresponding hadrons. They are usually defined in momentum space where they describe the probability amplitudes to find in a hadron with momentum $\left\{P^{+}, \mathbf{P}\right\}$ 円 and well defined angular momentum and flavor content a quark and an antiquark with momenta $\left\{z P^{+}, \mathbf{k}+z \mathbf{P}\right\}$ and $\left\{(1-z) P^{+},-\mathbf{k}+(1-z) \mathbf{P}\right\}$. One crucial property of light cone wave functions is their dependence on $z$ and $\mathbf{k}$ alone [18, 19.

\footnotetext{
${ }^{1}$ Light cone coordinates are $P^{ \pm}=\left(P^{0} \pm P^{3}\right) / \sqrt{2}, \mathbf{P}=\mathbf{P}_{T}=\left(P^{1}, P^{2}\right)$. A particle momentum is fully specified by the set $\left\{P^{+}, \mathbf{P}\right\}\left(P^{-}=\left(\mathbf{P}^{2}+M^{2}\right) / 2 P^{+}\right.$with $M$ the particle mass $)$.
} 
This implies that upon Fourier transformation in the transverse plane, the relative coordinate of the $q \bar{q}$ pair, $\mathbf{r}=\mathbf{x}_{q}-\mathbf{x}_{\bar{q}}$, is easily separated from the position of the hadron "center", $\mathbf{X}=z \mathbf{x}_{q}+(1-z) \mathbf{x}_{\bar{q}}$. In a similar way to non relativistic physics, the non trivial degree of freedom of the wave function can be isolated, the resulting transition matrix element is

$$
\left\langle z, \mathbf{x}_{q}, \mathbf{x}_{\bar{q}} \mid P^{+}, \mathbf{P}\right\rangle=\psi(z, \mathbf{r}) e^{i \mathbf{P} \cdot \mathbf{X}}
$$

In the amplitude given in Eq. (2.4) the impact parameter $\mathbf{b}$ denotes the transverse separation between hadron centers, $\mathbf{b}=\mathbf{X}_{1}-\mathbf{X}_{2}$. The transverse positions of quarks are then given by

$$
\begin{aligned}
& \mathbf{x}_{1}=\mathbf{x}_{0}+\mathbf{b} / 2+\left(1-z_{1}\right) \mathbf{r}_{1} \\
& \mathbf{x}_{2}=\mathbf{x}_{0}-\mathbf{b} / 2+\left(1-z_{2}\right) \mathbf{r}_{2}, \\
& \mathbf{x}_{0}=\left(\mathbf{X}_{1}+\mathbf{X}_{2}\right) / 2
\end{aligned}
$$

i.e. $\mathbf{x}_{0}$ is in the center of $\mathbf{X}_{1}$ and $\mathbf{X}_{2}$. Antiquark positions are related to quark positions as $\mathbf{x}_{\bar{\imath}}=\mathbf{x}_{i}-\mathbf{r}_{i}$. We notice that the reference point $\omega$ chosen in Sec. IIB "moves" with respect to $\mathbf{x}_{0}, \mathbf{x}_{\omega}=\mathbf{x}_{0}-1 / 2\left[\left(z_{1}-1 / 2\right) \mathbf{r}_{1}-\left(z_{2}-1 / 2\right) \mathbf{r}_{2}\right]$. We have, however, checked that choosing $x_{0}$ rather than $\omega$ as the reference point for the computation of the loop-loop amplitude has negligible numerical effects.

In the present study, we are interested in electroproduction of different vector mesons under various kinematical conditions on a fixed proton target. It is therefore instructive to isolate the variable part of the amplitude associated with the photon and vector meson coordinates, $z_{1}, \mathbf{r}_{1}$, by integrating out the nucleon coordinates, $z_{2}, \mathbf{r}_{2}$. To this end we define

$$
J_{p}\left(z_{1}, \mathbf{r}_{1}, \Delta_{T}\right)=2 \int_{0}^{+\infty} b d b 2 \pi J_{0}\left(\Delta_{T} b\right) \int \frac{d z_{2} d^{2} \mathbf{r}_{2}}{4 \pi}\left|\psi_{p}\left(z_{2}, \mathbf{r}_{2}\right)\right|^{2} J\left(b, z_{1}, \mathbf{r}_{1}, z_{2}, \mathbf{r}_{2}\right)
$$

so that the amplitude Eq. (2.4) is now written as

$$
\mathcal{M}=i s \int \frac{d z_{1} d^{2} \mathbf{r}_{1}}{4 \pi} \psi_{V}^{\dagger} \psi_{\gamma}\left(z_{1}, \mathbf{r}_{1}\right) J_{p}\left(z_{1}, \mathbf{r}_{1}, \Delta_{T}\right)
$$

The determination of parameters of the model of the stochastic vacuum can be made in different ways. We follow the method given in Ref. [2]. We use as input parameters the total proton-proton cross section at $\sqrt{s}=20 \mathrm{GeV}$, namely $\sigma_{p p}=35 \mathrm{mb}$ and the slope $B_{p p}=11.5 \mathrm{GeV}^{-2}$ of the $p-p$ elastic cross section. From lattice simulations [15], we take the mixing coefficient $\kappa=0.74$ and the curve relating gluon condensate and correlation length. The square of the proton quark-diquark wave function is taken in the simple form

$$
|\psi(z, r)|^{2}=4 \omega^{2} \delta(z-1 / 2) e^{-\omega^{2} r^{2}}
$$

As ouput we obtain the gluon condensate $\left\langle g^{2} F F\right\rangle=2.49 \mathrm{GeV}^{4}$, the correlation length $a=$ $0.346 \mathrm{fm}$ and the proton transverse radius $R_{p}=1.51 a=0.52 \mathrm{fm}$. The parameters are different from those of Ref. [2] where the influence of the non-confining term, $D_{1}$, in the correlator Eq. (2.1) was neglected, i.e. $D_{1}$ was set to 0 . The proton size is smaller than the transverse rms charge radius, $R_{T}=0.68 \mathrm{fm}$. Sea-quark contributions may enter into the form factor 
increasing the charge radius compared to the radius of the valence quarks. The same sea quarks or extra color dipoles may also enter in the cross section at higher energies where it is increasing with energy. New lattice simulations 20] try to isolate the perturbative contributions from the nonperturbative gluon fluctuations in a more precise way and may be incorporated together with a better treatment of the perturbative two-gluon exchange.

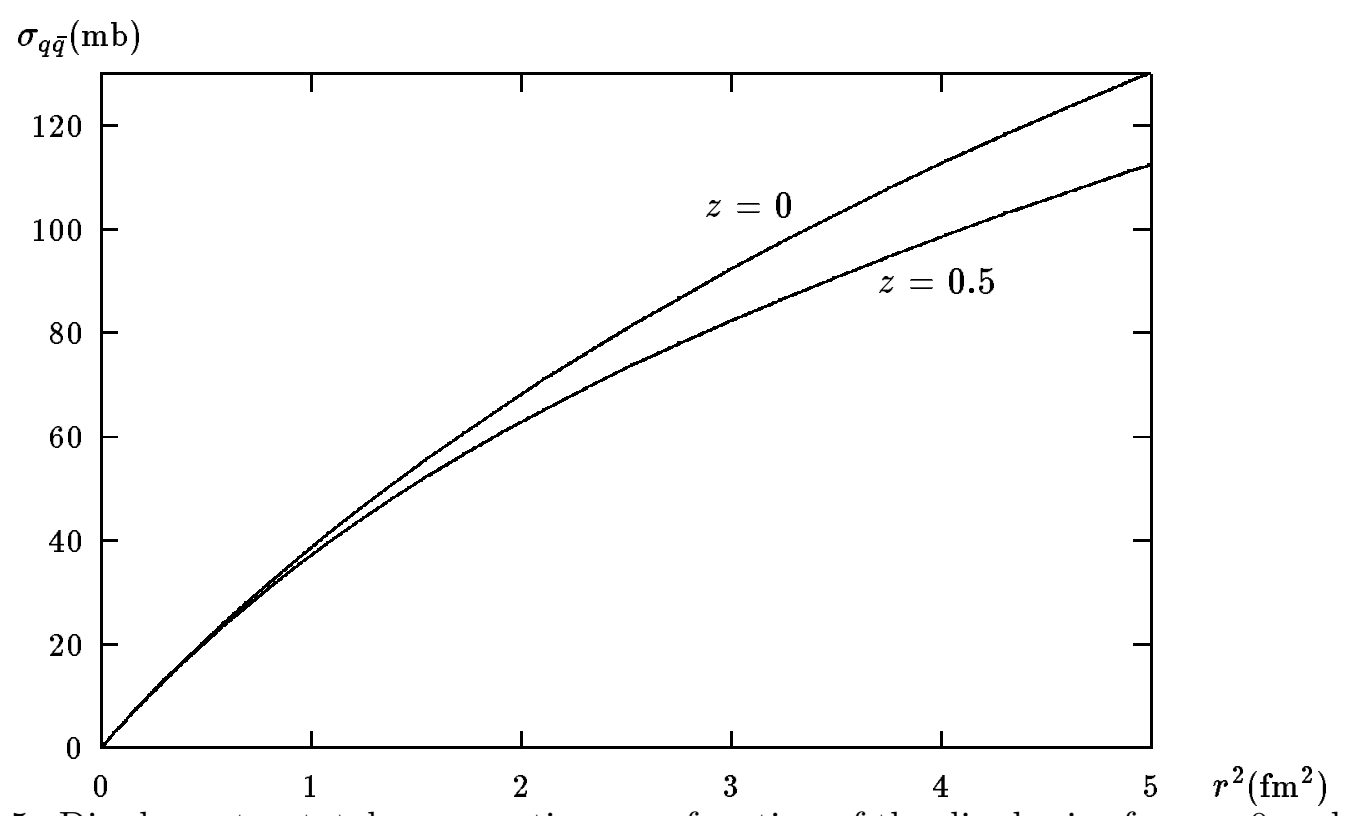

FIG. 5. Dipole-proton total cross section as a function of the dipole size for $z=0$ and $z=0.5$. The dependence in $z$ is rather marginal as it becomes noticeable only for very large separation of the $q \bar{q}$ pair. The cross section behaves as $\sigma_{q \bar{q}} \propto r^{n}$ with $n=2$ for small extension and slowly decreasing at larger distances.

In Fig. 5, we show the behavior of the function

$$
J_{p}^{(0)}\left(z, r, \Delta_{T}=0\right)=\int_{0}^{2 \pi} \frac{d \theta}{2 \pi} J_{p}\left(z, r, \theta, \Delta_{T}=0\right) .
$$

It represents the total cross section of a $q \bar{q}$ dipole of fixed size $r$ averaged over its orientation. For varying dipole sizes the total cross section on the proton increases quadratically for small dipoles until a size of $r=1-2 a$, then the increase continues but with a decreasing power. This feature is distinct to model of perturbative gluon exchange in Ref. [21] where this total cross section saturate at about twice the proton radius.

It is also instructive to consider the $\Delta_{T}$-fall off of the transition amplitude on the hadron radii and the correlation length $a$. In Ref. [2], the logarithmic slope $B$ of elastic cross sections has been numerically parametrized as

$$
B \approx 1.56 a^{2}+0.24\left(R_{1}^{2}+R_{2}^{2}\right)
$$

The large first term which is independent of the radii is specific for the model. 


\section{ELECTROPRODUCTION OF VECTOR MESONS}

In this section, we describe the $q \bar{q}$ wave functions of the photon and vector meson which enter into the expression of the production amplitude Eq. (2.6). Even at the qualitative level there are still large uncertainties about the correct dynamical description of hadrons. Indeed, one of the most interesting issues of current and future experiments is to shed light on the long distance properties of QCD. This evidently includes the unravelling of basic facts about hadronic wave functions. We shall show that the electroproduction of vector mesons is quite sensitive to their wave function.

For the time being, one has to make assumptions which influence the result as strongly as the dynamical features of the transition operator for diffraction given by the model of the stochastic vacuum. In the analysis of hadron-hadron scattering [2], a simple transverse wave function for the $\pi$ meson has been chosen

$$
\psi(r)=2 \omega e^{-\omega^{2} r^{2} / 2} .
$$

Note that in this reference the dependence of the geometry of the loop-loop interaction on the respective light cone fractions of the quarks and antiquarks was not yet considered. Including this dependence and wave functions $\psi(z, r)$ with a reasonable behavior on the light cone momentum fraction $z$, we found similar results to those given in Ref. [2]. The transverse size of the studied hadrons still determines the size of hadron-hadron cross sections. We shall see in the following how this feature is modified when one is considering photon induced reaction.

\section{A. Photon wave function}

The $q \bar{q}$ wave function of the photon carries as labels the virtuality $Q^{2}$ and the polarization state $\lambda$ of the photon. It describes the probability amplitude to find a quark-antiquark pair inside the photon with light cone fractions $(z, 1-z)$ and transverse separation $\mathbf{r}=$ $(r \cos \theta, r \sin \theta)$. The $q \bar{q}$ state is in a configuration with given flavor $(f, \bar{f})$ and helicities $(h, \bar{h})$. The color part of the wave function is treated separately and considered together with the Wilson loop in the way described in Sec. ПIB and we are only left here with an overall factor $\sqrt{N_{c}}$. The photon couples to the electric charge of the quarks with $e_{f} \delta_{f \bar{f}}$ where $e_{f}=2 / 3 e$ or $-1 / 3 e$ respectively. The helicity and spatial configuration part of the wave function looks different for various photon polarizations. It can be computed in light cone perturbation theory and one has to lowest order (see the Appendix A)

$$
\psi_{\gamma\left(Q^{2}, \lambda\right)}=\sqrt{N_{c}} e_{f} \delta_{f \bar{f}} \tilde{\psi}_{\gamma\left(Q^{2}, \lambda\right)},
$$

with

$$
\begin{aligned}
\tilde{\psi}_{\gamma\left(Q^{2}, 0\right)} & =-\delta_{h,-\bar{h}} 2 z(1-z) Q \frac{K_{0}(\varepsilon r)}{2 \pi}, \\
\tilde{\psi}_{\gamma\left(Q^{2}, 1\right)} & =\sqrt{2}\left(i e^{i \theta} \varepsilon\left(z \delta_{h+} \delta_{\bar{h}-}-(1-z) \delta_{h-} \delta_{\bar{h}+}\right) \frac{K_{1}(\varepsilon r)}{2 \pi}+m_{f} \delta_{h+} \delta_{\bar{h}+} \frac{K_{0}(\varepsilon r)}{2 \pi}\right), \\
\tilde{\psi}_{\gamma\left(Q^{2},-1\right)} & =\sqrt{2}\left(i e^{-i \theta} \varepsilon\left((1-z) \delta_{h+} \delta_{\bar{h}-}-z \delta_{h-} \delta_{\bar{h}+}\right) \frac{K_{1}(\varepsilon r)}{2 \pi}+m_{f} \delta_{h-} \delta_{\bar{h}-} \frac{K_{0}(\varepsilon r)}{2 \pi}\right),
\end{aligned}
$$




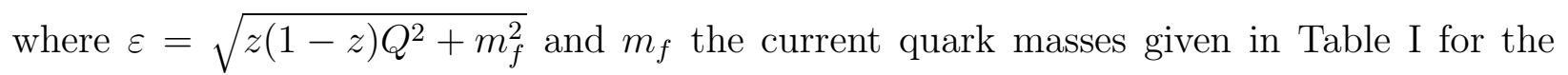
different flavors. $K_{0}, K_{1}$ are modified Bessel functions.

The longitudinal photon wave function is peaked around $z=1 / 2$, so that the longitudinal photon interacts like a small dipole, $r \sim 1 / Q$, at large $Q^{2}$. On the contrary, the transverse photon is almost flat in $z$ so that, at large $Q^{2}$, it interacts partly like a small object for intermediate $z$ and partly like a large one for $z \sim m_{f} / Q$ when light quarks are involved. For heavy quarks, $c, b$, the inverse of the quark mass limits the photon extension. In electroproduction of vector mesons the effective dipole size is fixed by the overlap of wave functions of the photon with the vector meson. Due to the shape of the latter the small $z$ region is somewhat suppressed and the transverse region explored for $Q$ above $1-2 \mathrm{GeV}$ is below or around $1 \mathrm{fm}$. Lacking a better knowledge in the region of large transverse size, we use the above wave function. This may be tested by forthcoming experiments on the ratio of longitudinal to transverse cross sections.

\section{B. Vector meson wave function}

For the hadron wave function, we use the same notation as described above for the photon. We have already taken care of color in the construction of the Wilson loops cf. Sec. IIB. For the flavor content we consider that the $\rho^{0}(770), \omega(782), \phi(1020)$ and $J / \psi$ mesons are respectively pure isospin 1 , isospin $0, s \bar{s}$ and $c \bar{c}$ vector mesons. This flavor part together with the configuration part $\tilde{\psi}$ forms the wave function $\psi_{V}$ needed in Eq. (2.6).

Some information has been obtained on the spatial dependence of hadron wave functions. The first piece of information comes from long distance physics which various quark models describe successfully. These models tell us that a hadron at rest can be modelled with Gaussian wave functions as a sytem of constituent quarks moving in a harmonic oscillator potential. To boost non relativistic wave functions to a fast moving system is not a trivial step. Technically the interplay between the transverse and longitudinal dynamics in light cone physics as well as the treatment of spin degrees of freedom remain to be understood. Within the model of the stochastic vacuum a nice quantitative description of hadron-hadron soft collisions has been obtained by disregarding spin and light cone fraction dependences. Therefore we assume that for soft collisions between large objects, a simple-minded description of hadrons Eq. (3.1) suffices. A smooth z-dependence on the light cone momentum fraction will not change this picture.

The second piece of information comes from short distance physics and perturbative QCD supplemented by sum-rules, where some properties of the valence wave function at 0 -transverse separation are known (in particular the end-point $z \rightarrow 0,1$ behavior can be analyzed). When wave functions at short distances are involved, e.g. in hard exclusive scatterings [22], the value of the meson wave function at the origin determines the value of cross sections. The wave function at the origin is related to the leptonic decay width of the meson.

The above observations allow to make an ansatz for vector meson wave functions:

$$
\tilde{\psi}_{V(0)}=z(1-z) \frac{\delta_{h,-\bar{h}}}{\sqrt{2}} \frac{\sqrt{2} \pi f_{V}}{\sqrt{N_{c}} \hat{e}_{V}} f(z) e^{-\frac{\omega^{2} r^{2}}{2}},
$$




$$
\begin{aligned}
\tilde{\psi}_{V(1)} & =\left\{\frac{i \omega^{2} r e^{i \theta}}{M_{V}}\left(z \delta_{h+} \delta_{\bar{h}-}-(1-z) \delta_{h-} \delta_{\bar{h}+}\right)+\frac{m_{f}}{M_{V}} \delta_{h+} \delta_{\bar{h}+}\right\} \frac{\sqrt{2} \pi f_{V}}{\sqrt{N_{c}} \hat{e}_{V}} f(z) e^{-\frac{\omega^{2} r^{2}}{2}}, \\
\tilde{\psi}_{V(-1)} & =\left\{\frac{i \omega^{2} r e^{-i \theta}}{M_{V}}\left((1-z) \delta_{h+} \delta_{\bar{h}-}-z \delta_{h-} \delta_{\bar{h}+}\right)+\frac{m_{f}}{M_{V}} \delta_{h-} \delta_{\bar{h}-}\right\} \frac{\sqrt{2} \pi f_{V}}{\sqrt{N_{c}} \hat{e}_{V}} f(z) e^{-\frac{\omega^{2} r^{2}}{2}} .
\end{aligned}
$$

This ansatz has the following properties. The main transverse dependence $e^{-w^{2} r^{2} / 2}$ and the function $f(z)$ are modeled in the way proposed by Wirbel, Stech and Bauer [23]

$$
f(z)=\mathcal{N} \sqrt{z(1-z))} e^{-M_{V}^{2}(z-1 / 2)^{2} / 2 \omega^{2}} .
$$

We use the same functional form for all vector mesons. The transverse size parameter $\omega$ is related to the vector meson radius, $\left\langle R_{3}^{2}\right\rangle^{1 / 2}=\left\langle x^{2}+y^{2}+z^{2}\right\rangle^{1 / 2}$, which for the Gaussian shape adopted is $\left\langle R_{3}^{2}\right\rangle^{1 / 2}=\sqrt{3 / 2} / 2 \omega$. This quantity is presumably not very different from the electromagnetic radius which unfortunatly is unknown for vector mesons. The way out in the quark model is to fix $\omega$ and $\mathcal{N}$ by the normalization and the $e^{+} e^{-}$decay width (see Appendix B). We draw attention to the fact that applying this procedure to the above parametrization of the wave function leads to different sets of parameters, $\{\omega, \mathcal{N}\}$, for longitudinal and transversal mesons. We find radii in this way which are reasonable in the whole family of vector mesons. This is a welcome property because, as we shall see in Sec. IV, the vector meson transverse size is one of the important ingredients determining the cross sections in the intermediate $Q^{2}$-range. The above form is written to have explicitly the correct value of the wave function at the origin, i.e. the $q \bar{q}$-state fulfills the equation

$$
\left\langle 0\left|J^{\mu}(0)\right| V(q, \lambda)\right\rangle=e f_{V} M_{V} \varepsilon^{\mu}(q, \lambda)
$$

with $f_{V}$ the meson decay constant. In Eq. (3.3), $\hat{e}_{V}$ is the mean quark charge in the meson

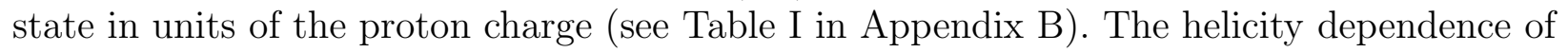
the wave functions is modeled after the perturbative $\gamma \rightarrow q \bar{q}$ transition.

\section{Cross sections}

Let us collect the formula necessary to compute cross sections. It is convenient to expand the quantity $J_{p}$ in Eq.(2.5) in terms of $L_{z}$-eigenfunctions

$$
J_{p}\left(z_{1}, r_{1}, \theta_{1}, \Delta\right)=\sum_{m} e^{i m \theta_{1}} J_{p}^{(m)}\left(z_{1}, r_{1}, \Delta\right)
$$

where thanks to the periodicity of $J_{p}$ only even $m$ are present. It follows that in the reaction studied, only transverse to transverse and longitudinal to longitudinal transitions are expected. From the explicit form of the wave function, Eqs. (3.2) and (3.3), there is a possibility of helicity change by two units in the process. We have, however, observed that the corresponding contribution to the cross section is smaller than $2 \%$ in the whole $Q^{2}$-range. We disregard this contribution.

In the following, we distinguish transverse and longitudinal cross sections which, in the present conventions, are 


$$
\begin{aligned}
\frac{d \sigma_{L}}{d t} & =\frac{1}{16 \pi}\left|\int \frac{d z_{1} r_{1} d r_{1}}{2} \psi_{V(0)}^{\dagger} \psi_{\gamma(0)}\left(z_{1}, r_{1}\right) J_{p}^{(0)}\left(z_{1}, r_{1}, \Delta\right)\right|^{2} \\
\frac{d \sigma_{T}}{d t} & =\frac{1}{16 \pi}\left|\int \frac{d z_{1} r_{1} d r_{1}}{2} \psi_{V(1)}^{\dagger} \psi_{\gamma(1)}\left(z_{1}, r_{1}\right) J_{p}^{(0)}\left(z_{1}, r_{1}, \Delta\right)\right|^{2},
\end{aligned}
$$

where the average over proton helicities is understood. The combination $\psi_{V(\lambda)}^{\dagger} \psi_{\gamma(\lambda)}\left(z_{1}, r_{1}\right)$ are computed by multiplying Eq. (3.2) by Eq. (3.3) supplemented by the flavor part described in Sec. IIIB giving

$$
\begin{aligned}
& \psi_{V(0)}^{\dagger} \psi_{\gamma(0)}=-e f_{V} z_{1}\left(1-z_{1}\right) f\left(z_{1}\right) e^{-\omega^{2} r_{1}^{2} / 2} 2 z_{1}\left(1-z_{1}\right) Q K_{0}\left(\varepsilon r_{1}\right) \\
& \psi_{V(1)}^{\dagger} \psi_{\gamma(1)}=e f_{V} f\left(z_{1}\right) e^{-\omega^{2} r_{1}^{2} / 2}\left\{\frac{\omega^{2} \varepsilon r_{1}}{M_{V}}\left[z_{1}^{2}+\left(1-z_{1}\right)^{2}\right] K_{1}\left(\varepsilon r_{1}\right)+\frac{m_{f}^{2}}{M_{V}} K_{0}\left(\varepsilon r_{1}\right)\right\}
\end{aligned}
$$

Experimentally, the differential cross sections Eq. (3.5) are difficult to measure. On the one hand, the separation of transverse and longitudinal cross section is not easily done, on the other hand accurate data exist only for $t$-integrated cross section. Nevertheless, some results have been obtained for

$$
\frac{d \sigma_{\exp }}{d t}=\epsilon \frac{d \sigma_{L}}{d t}+\frac{d \sigma_{T}}{d t}
$$

as a function of $\Delta_{T}^{2} . \epsilon$ is the rate of longitudinally polarized photons which depends on the lepton scattering angle, $\theta_{e}$, and the photon energy, $\nu$. In the proton rest frame

$$
\epsilon=\left[1+2\left(1+\nu^{2} / Q^{2}\right) \tan ^{2}\left(\theta_{e} / 2\right)\right]^{-1}
$$

We shall also compare our theoretical results with the integrated cross section $\sigma_{\exp }=\epsilon \sigma_{L}+$ $\sigma_{T}$, for various $Q^{2}$. By analyzing the vector meson decay, it is possible to check the validity of $s$-channel helicity conservation or assuming helicity conservation to deduce $R=\sigma_{L} / \sigma_{T}$.

\section{NUMERICAL INVESTIGATION}

\section{A. General results}

Before entering in a detailed analysis, let us discuss qualitatively the $Q^{2}$-dependence of differential cross section expected from Eq. (3.5). The quantity $J_{p}^{(0)}\left(z_{1}, r_{1}, t\right)$ is power behaved in $r_{1}$ and decreases exponentially with $|t|$. It depends only weakly on $z_{1}$. At fixed $t$, $J_{p}^{(0)} \propto r_{1}^{\alpha}$ with $\alpha=2$ at small $r_{1}$ and $\alpha$ slowly decreasing for $r_{1} \gtrsim a$ (see Fig. 5). Given this behavior, we also need an estimate of the effective size of the photon-vector meson overlap after integration over $z_{1}$ in Eq. (3.6). One can anticipate two extreme regimes. At small $Q^{2}$, the effective size is driven by the meson wave function so that $d \sigma_{L} \propto Q^{2}$ and $d \sigma_{T}$ is constant. At large $Q^{2}$, the effective size is given by the photon wave function alone, which leads to $d \sigma_{L} \propto Q^{-6}$ and $d \sigma_{T} \propto Q^{-8}$.

These asymptotic behaviors of cross section are rather model-independent. Most of the current experimental results, however, are below the asymptotic large $Q^{2}$-region. The theoretical behavior of cross sections in our calculation shows a much more specific dependence on $Q^{2}$ in this region. In Fig. 6] we show the logarithmic $\rho$-production cross section 


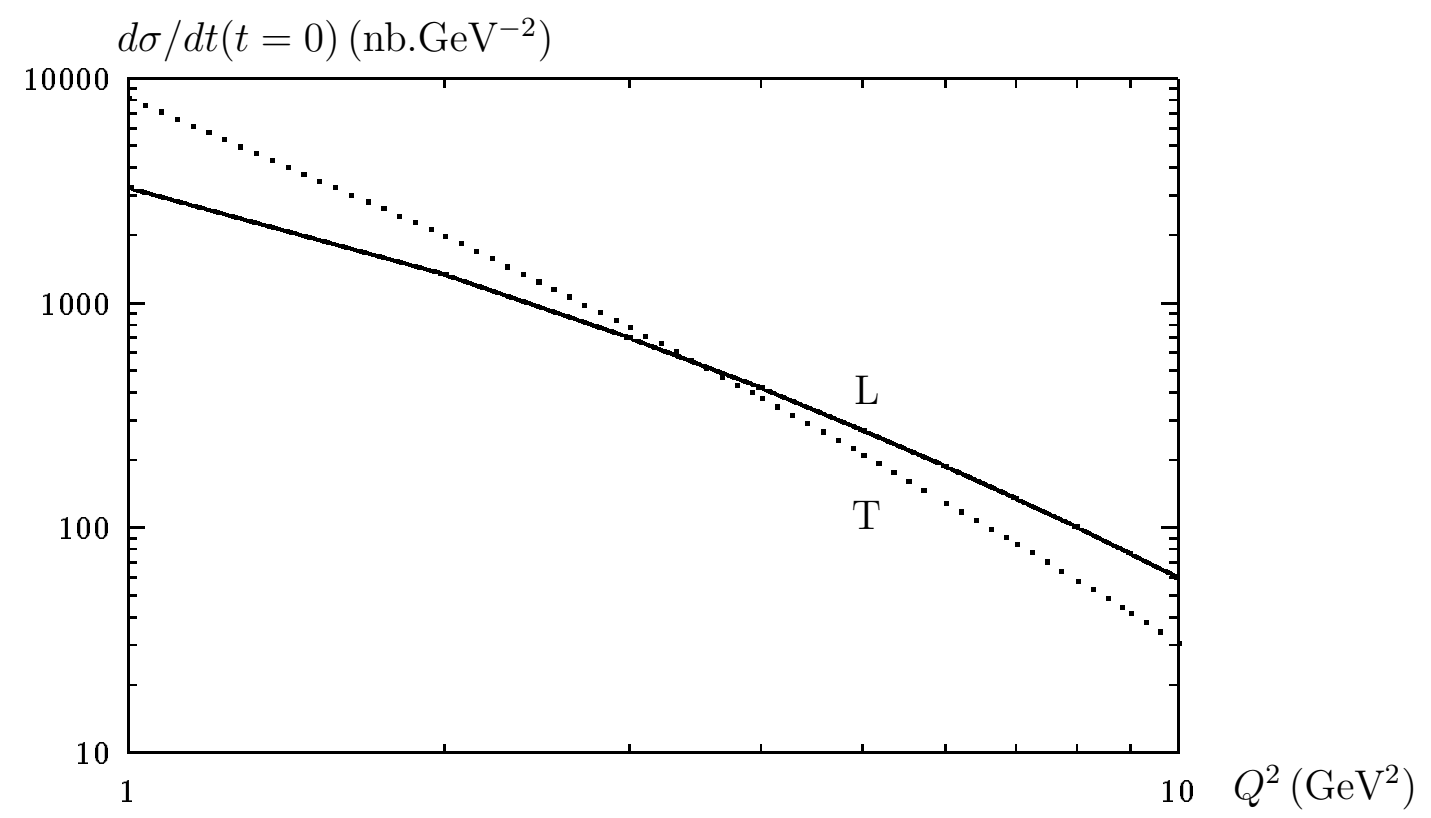

FIG. 6. $d \sigma / d t(t=0)$ for $\rho$-production as a function of $Q^{2}$ for the longitudinal (full) and transverse (dots) cross section. The effective power of the fall off with $Q$ is increased by 2 units in the range $1-10 \mathrm{GeV}^{2}$ but the asymptotic behavior is only reached in the $10-100 \mathrm{GeV}^{2}$

$\log d \sigma / d t(t=0)$ as a function of $Q^{2}$. The effective power $n$ of the fall off $d \sigma / d t(t=0) \propto Q^{-n}$ is about 2.5 and 4 at $Q^{2}=1 \mathrm{GeV}^{2}$ for the longitudinal and transversal cross section respectively and this power increases to about 4.5 and 6 respectively at $Q^{2}=10 \mathrm{GeV}^{2}$. Thus the asymptotic regime starts only above that value.

As we have emphasized the result depends on the wave function chosen to represent the vector meson state. Without changing the parametrization, this can be seen by varying the size parameter $\omega$ of the vector meson wave function. With the value of the wave function at the origin kept fixed we observe that decreasing $\omega$ by $5 \%$ increases the cross section at $Q^{2}=1 \mathrm{GeV}^{2}$ by about $20 \%$. This modification becomes less than $3 \%$ at $Q^{2}=10 \mathrm{GeV}^{2}$ because the vector meson size is less important for a small $q-\bar{q}$ state in the photon. One can also think of changing the light cone distribution of the quark and antiquark in the meson wave function. An indication of the resulting modification is given by changing the factor $\sqrt{z(1-z)} \rightarrow[z(1-z)]^{3 / 2}$ in the parametrization of $f(z)$ in Eq. (3.4). This leads to about $30 \%$ decrease of the cross section in the whole $Q^{2}$ range examined.

In Fig. 7, we show the importance of the transversal extension of the virtual photon. We introduce a transversal cut-off in the cross section, i.e. we cut the $r_{1}$-integration in Eq. (2.6) at a fixed value $r_{1} \leq r_{1}^{\text {cut }}$. As expected the $q \bar{q}$-wavefunction in a transversal photon extends to much larger values of $r_{1}$, e.g. at $Q^{2}=4 \mathrm{GeV}^{2}$ more than $50 \%$ of the cross section comes from transverse separations bigger than $1 \mathrm{fm}$. In the longitudinal case, the contribution of the region with transverse separations $r_{1}>1 \mathrm{fm}$ drops down to $15 \%$ at $Q^{2}=4 \mathrm{GeV}^{2}$. 

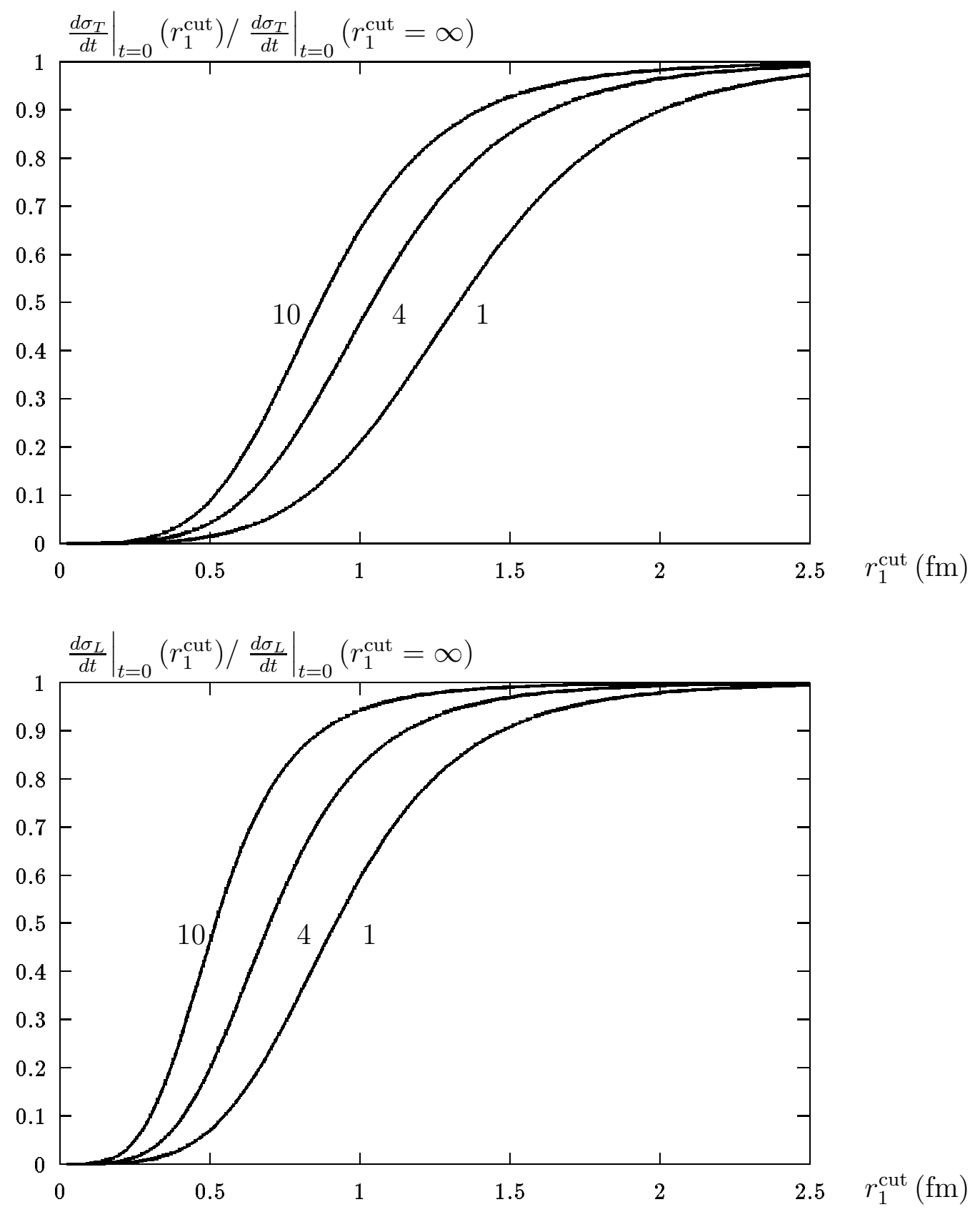

FIG. 7. (a) The contribution to the transverse differential cross section of $\rho$-production with respect to the radial parameter of the photon and vector meson side. The integration over the transverse distance $r_{1}$ is taken up to a cut-off $r_{1}^{\text {cut }}$ for several values of the photon virtuality, $Q^{2}=1,4$ and $10 \mathrm{GeV}^{2}$. The transverse differential cross section at $t=0$ is plotted as a function of $r_{1}^{\text {cut }}$. It is normalized to its value with the cut-off removed. (b) Same as (a) for longitudinal polarization. With increasing $Q^{2}$ the cross section is dominated by short transverse distances in both cases but the saturation occurs earlier for the longitudinal cross section. 


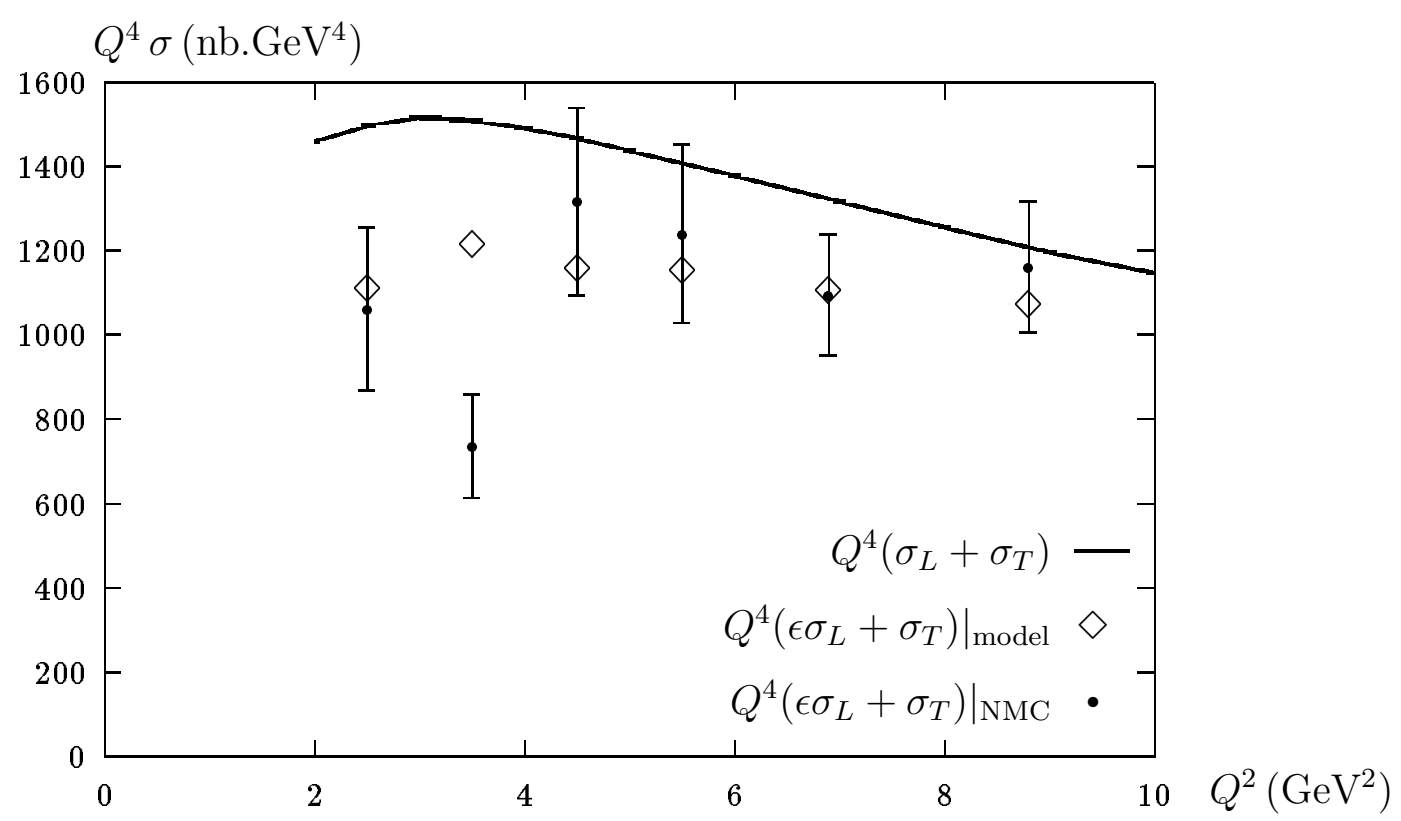

FIG. 8. The scaled cross section $Q^{4} \sigma\left(Q^{2}\right)$ for $\rho$-production in $n b \cdot \mathrm{GeV}^{4}$. The circles are the NMC-results [24] and the diamonds represent our prediction for the quantity $Q^{4}\left(\epsilon \sigma_{L}+\sigma_{T}\right)$ with the experimental polarization rate of NMC.

\section{B. $\rho, \omega, \phi$-production}

In the range from 1 to $10 \mathrm{GeV}^{2}$, the $1 / Q^{4}$ behaviour of the production cross section observed by EMC and NMC is very well reproduced by our calculations. Besides the $Q^{2}$ dependence, also the absolute values of the cross section are reproduced. It should be noted that within our model we have introduced no new parameters and the parameters underlying the interaction on the quark-gluon level are determined by soft high-energy proton(anti)proton scattering. We show in Fig. 8 NMC deuteron data [24] together with our prediction for $Q^{4}\left(\sigma_{L}+\sigma_{T}\right)$. We notice that this theoretical quantity is not the actually measured cross section due to the polarization rate of the photon, $\epsilon \neq 1$. Therefore we also show with diamonds the quantity $Q^{4}\left(\epsilon \sigma_{L}+\sigma_{T}\right)$ at the $Q^{2}$-points of NMC using their value of $\epsilon\left(Q^{2}\right)$.

In our approach, the approximate $1 / Q^{4}$ behavior is due to a combination of different fall offs in $\sigma_{L}$ and $\sigma_{T}$ which themselves come from the interplay between the size dependence of the dipole-proton cross section and the effective size of the photon-meson overlap. This is very different from the dynamics which occur in quark-quark scattering which leads however to a similar $Q^{2}$-dependence. As explained above the asymptotic behaviour for large $Q^{2}$ is just governed by the dipole size of the virtual photon and thus model independent.

A possible way to distinguish both approaches would be a precise measurement of the $Q^{2}$-behavior of the ratio of longitudinal to transverse cross sections $R\left(Q^{2}\right)=\sigma_{L} / \sigma_{T}$. We plot this ratio in Fig. 9. For large $Q^{2}, R \propto Q^{2}$ but this behavior is not yet reached in the intermediate range where $R$ grows slower than $Q^{2}$. Here we expect a rather different behavior in our model compared to other models.

Another important check is provided by looking at the $p_{T}$-dependence of the differential cross section. We show our result for $\epsilon d \sigma_{L} / d t+d \sigma_{T} / d t$ versus $\Delta_{T}^{2}$ at $6 \mathrm{GeV}^{2}$ and compare in Fig. 10 to the NMC points for the deuteron outside of the coherent production region [24]. 


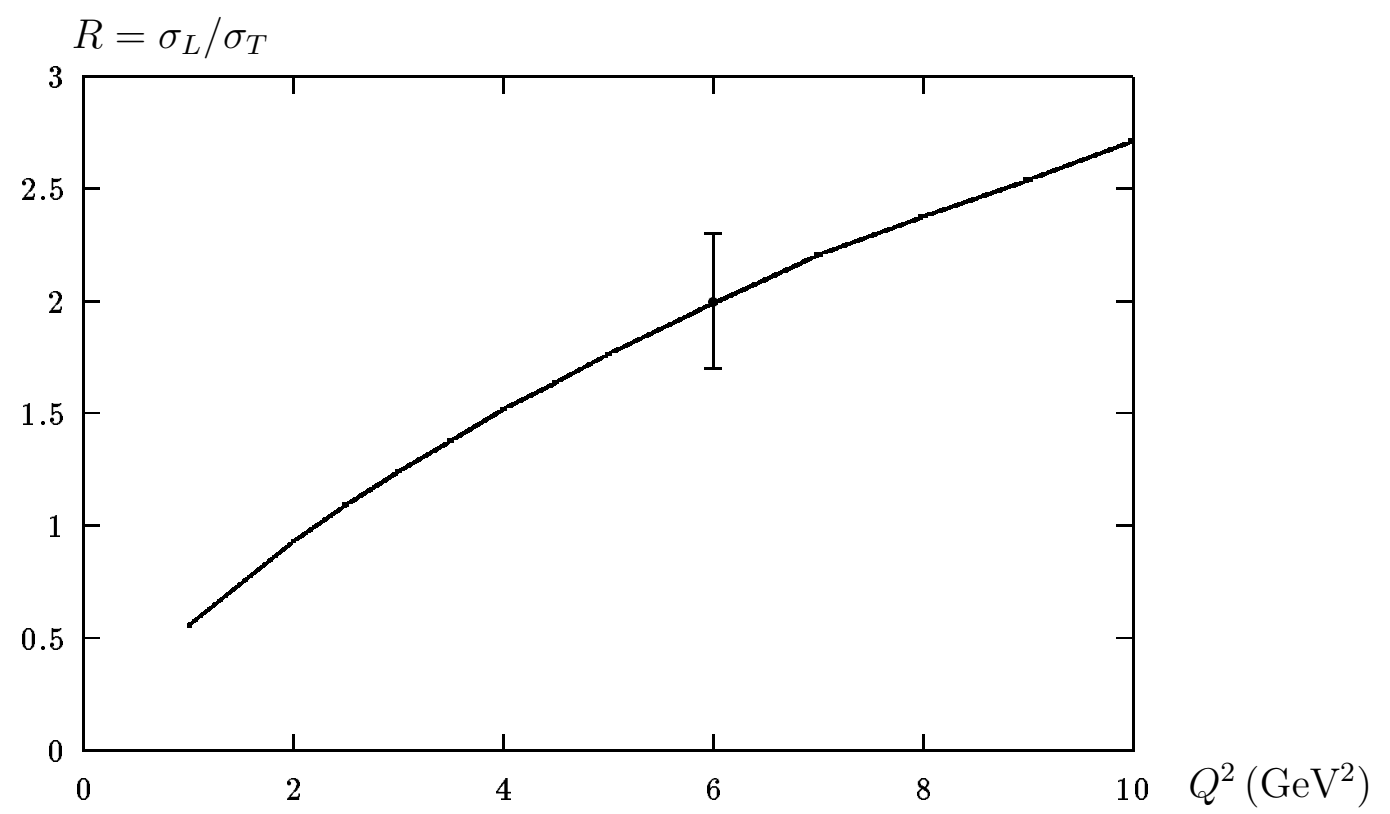

FIG. 9. The ratio for longitudinal to transverse cross section for $\rho$-production. The data point is from Ref. 24]. Other data compare well within errors but they either are far outside the $10-20 \mathrm{GeV}$ range or have large errorbars.

Notice that the $t$-dependence of our computation is not fully exponential.

There is a non trivial $Q^{2}$-dependence of the slope $B$ in our model which is due to a decreasing transverse region probed by the slowly shrinking size of the photon as $Q^{2}$ grows. We show this in Fig. 11.

For the production of the $\omega$-meson, we expect a wave function very similar to the one of the $\rho$ and correspondingly the ratio should be determined through the flavour factor

$$
f_{\omega}^{2} / f_{\rho}^{2} \approx 9 \%
$$

Indeed, this is observed.

The situation is more complex for the production of heavier vector mesons where the different quark content has several consequences. The direct appearance of a mass term in the meson wave function gives an additionnal component in the overlap Eq. (3.6) and also modifies the photon extension parameter $\varepsilon^{2}=z(1-z) Q^{2}+m_{f}^{2}$. The quark content also influences both the transversal size of the vector meson and its momentum fraction distribution $f(z)$. Whereas the first effects are quite easily controlable, nothing precise is known about the quantitative effect of a heavier mass on the distribution $f(z)$. Qualitatively, one expects that the distribution becomes more peaked at $z=1 / 2$ as the mass of the constituents increases.

For $\phi$-meson, a smaller transversal extension than in the $\rho$-meson is expected due to the heavier $s$-quark mass. The smaller size of the $\phi$-meson reduces the $\phi$-production cross section at low $Q^{2}$ values beyond the flavour factor

$$
f_{\phi}^{2} / f_{\rho}^{2} \approx 27 \%
$$

Such an effect is also observed in the difference between pion-nucleon and kaon-nucleon scattering in the model [2]. It should diminish with increasing $Q^{2}$ in electroproduction since 


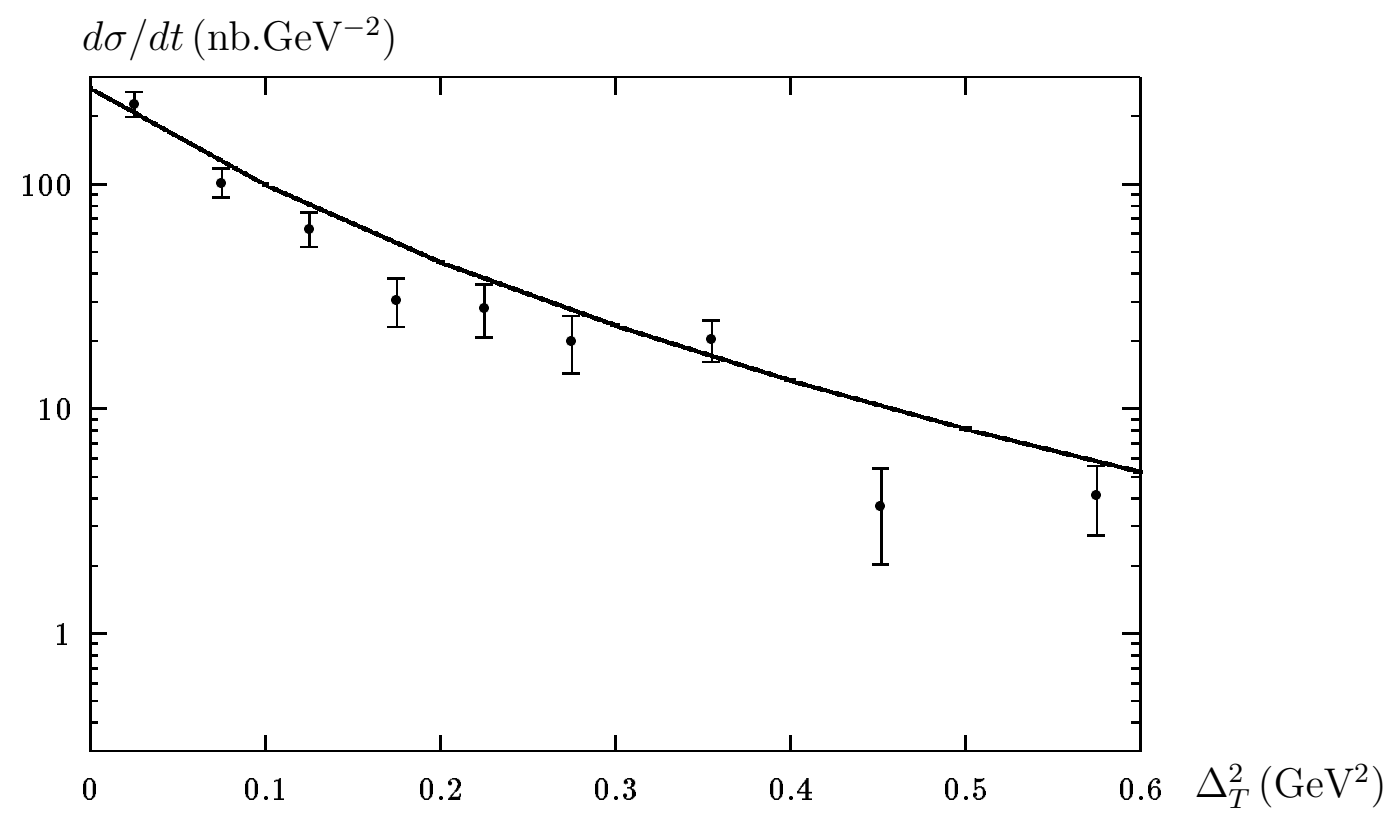

FIG. 10. The differential cross section, $d \sigma / d t\left(\Delta_{T}^{2}\right)$, for $\gamma^{*}+p \rightarrow \rho+p$ at $Q^{2}=6 \mathrm{GeV}^{2}$. Data from Ref. [24].

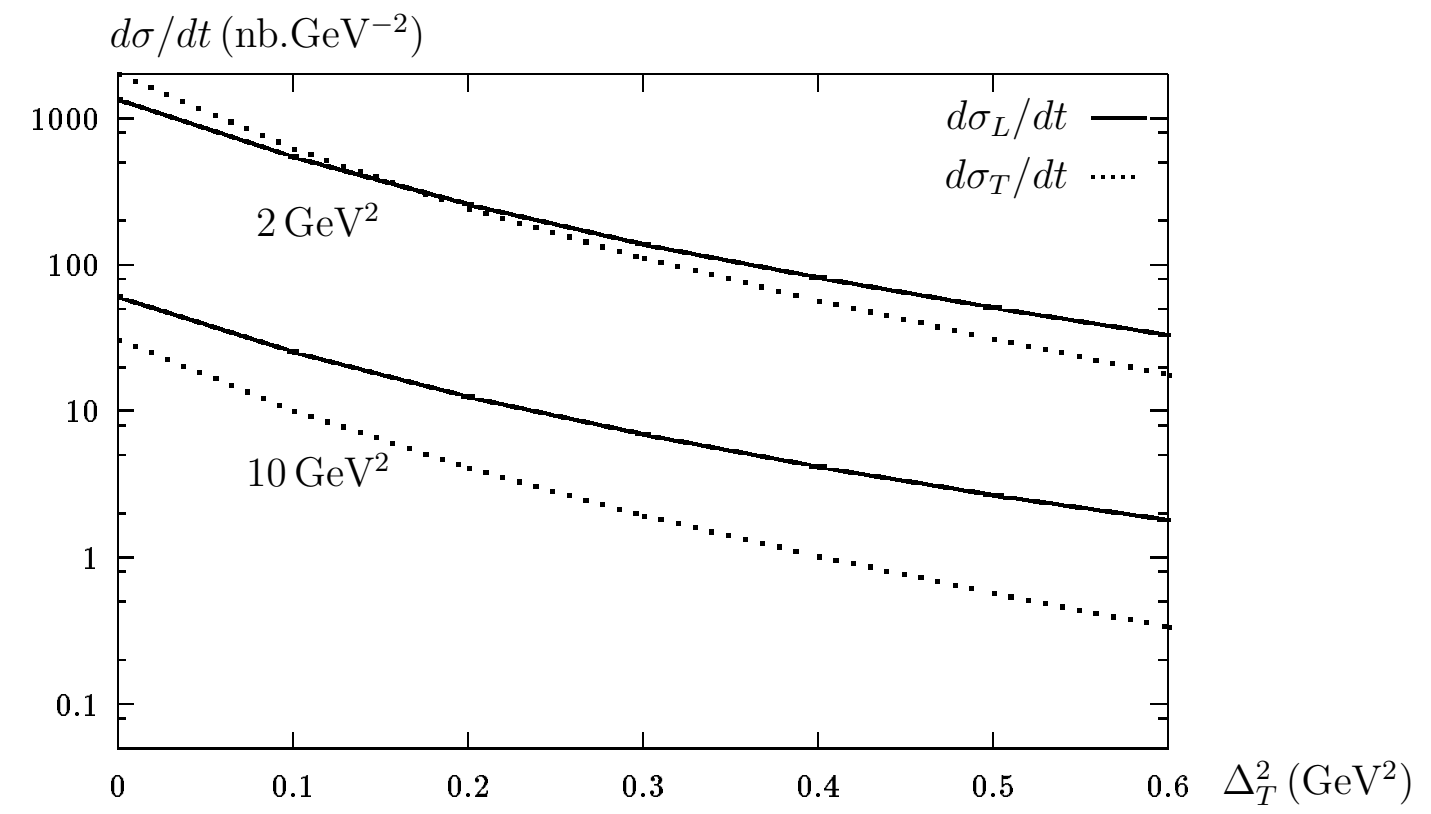

FIG. 11. The differential cross section for $\rho$-production, $d \sigma / d t\left(\Delta_{T}^{2}\right)$, for a longitudinal photon (full lines) and a transverse one (dotted lines) at $Q^{2}=2$ and $10 \mathrm{GeV}^{2}$. 


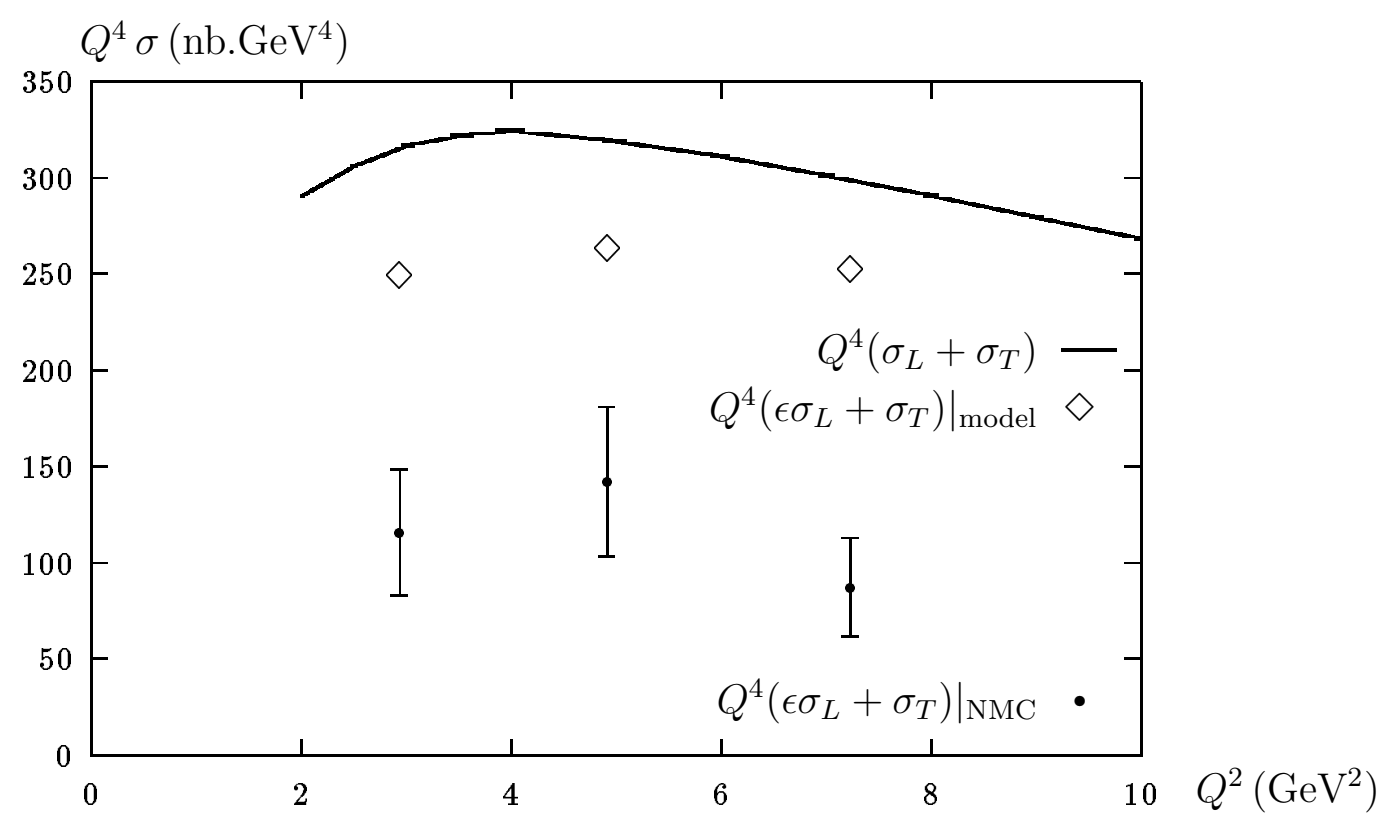

FIG. 12. $\phi$-production cross section compared to NMC data 24]. In our model the difference between theoretical and experimental result is attributed to wave function effect.

then the amplitude is less and less determined by the extension of the produced meson, but rather by the virtual photon. This effect may have been observed in the ZEUS data [25]. Also the change of the longitudinal distribution in $z$ between $\phi$ and $\rho$-meson may influence the cross section independently of $Q^{2}$. This difference is absent with our choice of distribution for the $\phi$-meson which is numerically the same as for the $\rho$-meson. Our resulting theoretical cross section for the $\phi$-production reproduce the $Q^{2}$ dependence of the NMC data, but its absolute value is practically a factor 2 too large (see Fig. 12).

The increase of the longitudinal to transverse ratio, $R\left(Q^{2}\right)$, looks the same as for the $\rho$-meson, its overall magnitude being just reduced by about $20 \%$. The $p_{T}$-dependence also exhibits a similar pattern with a small broadening of the diffraction peak as $Q^{2}$ is increased.

\section{C. $J / \psi$-production}

For heavier quark pairs, the large quark mass leads to more dramatic modifications. Let us first notice that in our model we assume that the quarks move on lightlike trajectories. This can only be the case at energies far above $2 m_{f}$. Therefore a center-of-mass energy bigger than $10 \mathrm{GeV}$ is necessary in the $J / \psi$-case. At these energies a moderate energy dependence is observed which is not contained in our model. In return, the large quark mass provides a hard scale so that also photoproduction data are accessible within our perturbative treatment of the photon. Strictly speaking, the difference $t_{0}=-M_{p}^{2}\left(Q^{2}+M_{V}^{2}\right)^{2} / s^{2}$, between $t$ and $-\Delta_{T}^{2}$, leads to a phase space threshold, $e^{B t_{0}}$, for the $J / \psi$-production in the energy range we are considering. At $\sqrt{s}=15 \mathrm{GeV}$, it is easy to see that this threshold effect is only sizeable for large $Q^{2}$, e.g. for $Q^{2}=10 \mathrm{GeV}^{2}$ and $B=5-10 \mathrm{GeV}^{-2}$ one gets $e^{B t_{0}}=0.97-0.94$. We shall disregard this factor in the following.

The discussion of the asymptotic regime given in Sec. IVA can be refined in the presence of a large quark mass. Let us reexamine the short distance regime in the presence of the mass- 


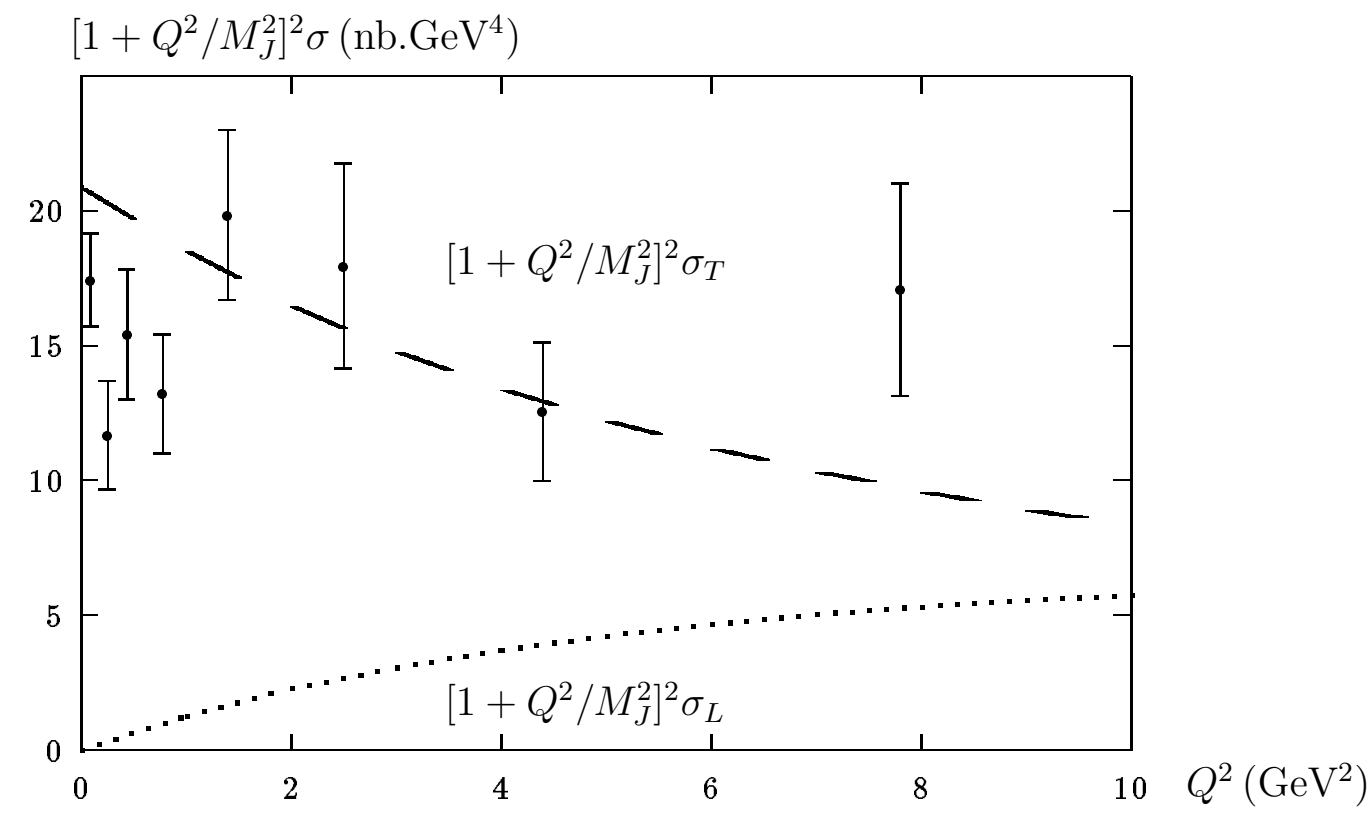

FIG. 13. $J / \psi$-production cross section for longitudinal (dots) and transverse (dashes) polarizations. To compare with EMC-data [26], one has to combine these two cross sections into $\sigma=\epsilon \sigma_{L}+\sigma_{T}$ with the polarization rate measured by EMC, $\epsilon \approx 0.7$.

terms in Eq. (3.6). To simplify further, we temporarily assume a simple non-relativistic form for the distribution, i.e. $f(z) \propto \delta(z-1 / 2)$ and for consistency take $m_{c}=M_{J} / 2$. We consider the domain of large enough $\varepsilon^{2}=m_{c}^{2}+Q^{2} / 4=\left(M_{J}^{2}+Q^{2}\right) / 4$, where we can approximate $\exp \left(-\omega^{2} r^{2} / 2\right) \approx 1$ and $J_{p}^{(0)}\left(z_{1}=1 / 2, r_{1}, \Delta=0\right) \approx C r_{1}^{2}$. One gets

$$
\begin{aligned}
& \frac{d \sigma_{L}}{d t}(t=0)=\alpha_{\mathrm{em}}\left(8 f_{J} C\right)^{2} \frac{Q^{2}}{\left(M_{J}^{2}+Q^{2}\right)^{4}} \\
& \frac{d \sigma_{T}}{d t}(t=0)=\alpha_{\mathrm{em}}\left(8 f_{J} C\right)^{2} \frac{M_{J}^{2}}{\left(M_{J}^{2}+Q^{2}\right)^{4}}\left(\frac{M_{J}^{2}+8 \omega^{2}}{M_{J}^{2}+2 \omega^{2}}\right)^{2} .
\end{aligned}
$$

From these expressions one sees that the relevant scale for $J / \psi$-production is $M_{J}^{2}+Q^{2}$ rather than $Q^{2}$. As in the light quark case, the longitudinal cross section is expected to dominate at large $Q^{2}$, namely the quantity $\left(M_{J}^{2}+8 \omega^{2}\right)^{2} /\left(M_{J}^{2}+2 \omega^{2}\right)^{2} \approx 1.4$ leads to a ratio $R \approx 0.7 Q^{2} / M_{J}^{2}$. The differential cross section is expected to fall off as $d \sigma / d t \propto(1+$ $\epsilon R)\left(M_{J}^{2}+Q^{2}\right)^{-4}$. Experimentally a $Q^{2}$-dependence such as $\left(M_{J}^{2}+Q^{2}\right)^{-n}$ is observed with $n$ around 2 but the accuracy of the data is not sufficient to exclude a complete short distance fall off. Let us stress that an accurate test of this power-law is a necessary prerequisite to understand the physics at work.

In Fig. 13, we plot $\left(1+Q^{2} / M_{J}^{2}\right)^{2} \sigma$, i.e. the cross section rescaled by the observed data fall off, for the transversal and longitudinal cross section separately together with the data recorded by EMC in the energy range $\sqrt{s}=10-20 \mathrm{GeV}$ [26]. The photoproduction comes out fairly in the energy range $\sqrt{s}=10-20 \mathrm{GeV}$, where several measurements have been performed leading to a production cross section between 10 and $20 \mathrm{nb}$. As could be guessed from the study of the asymptotic behavior above, the shape of the $z$-distribution and the value of the charm quark mass determine the size of the cross section. Changing the quark mass by $5 \%$ would lead to a $20 \%$ change in the cross section at $Q^{2}=0$ and to a $10 \%$ change at 


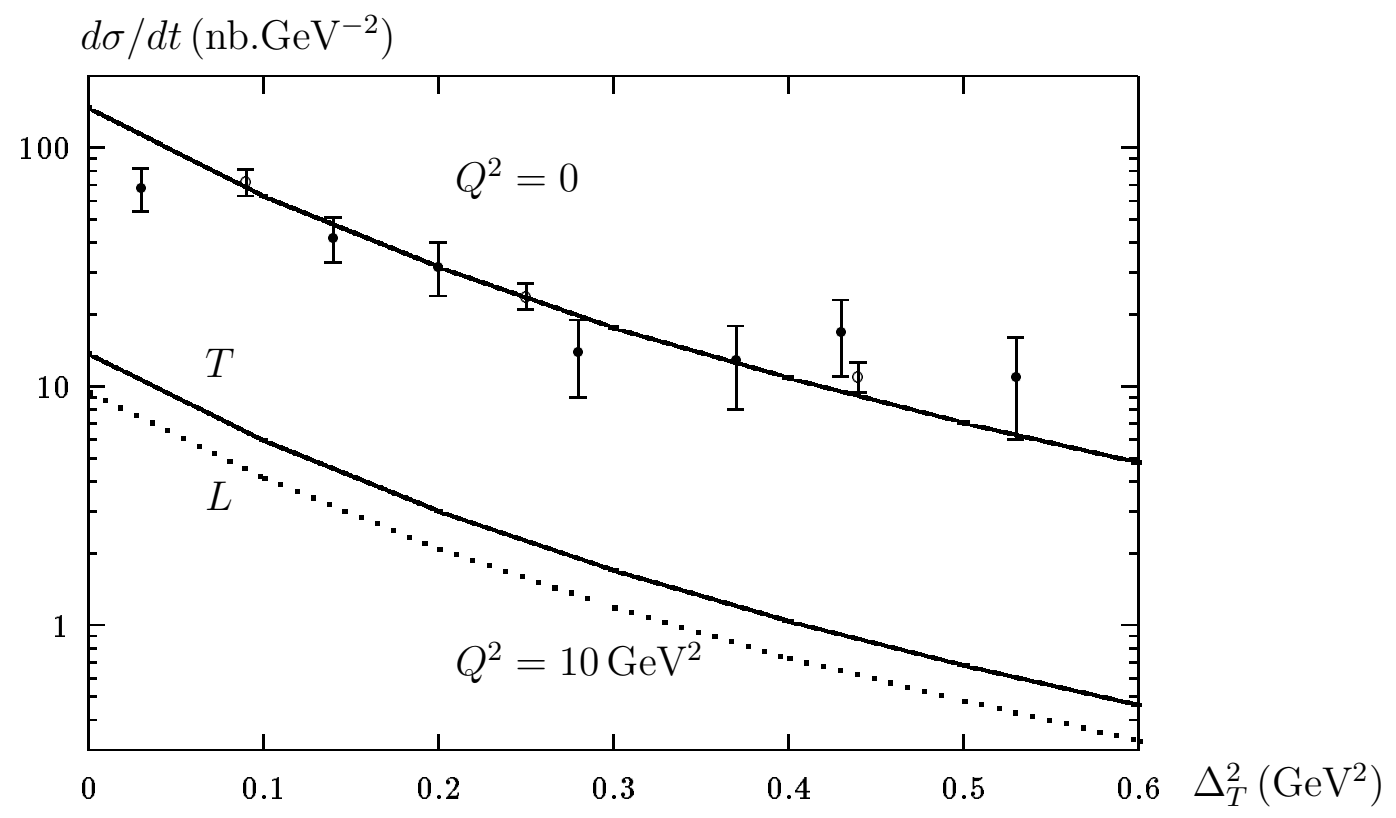

FIG. 14. $d \sigma / d t\left(\Delta_{T}^{2}\right)$ for $J / \psi$-production at $Q^{2}=0$ and $10 \mathrm{GeV}^{2}$. The upper curve is our prediction for the photoproduction differential cross section. It can be compared to the measurement of Ref. [27] (filled circles) and to the extrapolation to $Q^{2}=0$ of the EMC data [26]. Similar data have been measured by NMC [28] at $Q^{2}=1.5 \mathrm{GeV}^{2}$. Also shown are the differential cross sections at $Q^{2}=10 \mathrm{GeV}^{2}$ for longitudinal (dotted line) and transverse (lower full line) photons. The dependence of the slope on $Q^{2}$ and polarization is marginal in the $J / \psi$-case.

$Q^{2}=10 \mathrm{GeV}^{2}$ respectively. As $Q^{2}$ increases, our expectation follows qualitatively the pattern depicted for the short distance regime although quantitatively the intermediate transverse distance somewhat contributes to give a fall off flatter than the short distance one.

We next turn to the study of the $p_{T}$-dependence shown in Fig. 14. We find a good agreement with the photoproduction measurement [27] and with the extrapolation of EMC (open circles). We also note that, contrarily to the "large" hadron case, there is practically no $Q^{2}$-dependence of the $p_{T}$-fall off in the $J / \psi$-case.

\section{CONCLUSION}

We have calculated the longitudinal and transversal differential cross sections for diffractive production of $\rho, \omega, \phi$ and $J / \psi$-mesons in the range of $2 \mathrm{GeV}^{2} \leq Q^{2} \leq 10 \mathrm{GeV}^{2}$. The hadronic part of our calculation is based on a model for nonperturbative QCD, the model of the stochastic vacuum [1]. The parameters of the model which gives a unified description of low energy and soft high-energy scattering phenomena can be obtained from a variety of sources: hadron spectroscopy, QCD sum rules, high-energy proton-proton scattering and lattice calculations of the fundamental gluon field correlator. We have used a consistent parameter set very similar to the one used for hadron-hadron scattering in Ref. [2]. The virtual photon and hadron wave functions are light cone wave functions motivated by perturbation theory for the photon and relativistic quark models for the hadrons, we thus do not have adjustable parameters. A specific feature of electroproduction is the dependence of the cross sections on the photon virtuality $Q^{2}$ which is reproduced by the model almost perfectly. 
Even at $Q^{2}=10 \mathrm{GeV}^{2}$ the cross sections have not yet reached their asymptotic $1 / Q^{6}$ behaviour. Our calculation is consistent with the observed ratio of longitudinal to transverse cross sections. Here precise data at different values of $Q^{2}$ could discriminate between different models. In our model, we also can calculate the dependence on the tranverse momentum transfer and it nicely reproduces the available data.

Depending on photon polarization, the $Q^{2}$ range $2-10 \mathrm{GeV}^{2}$ corresponds to an effective transverse $q \bar{q}$ size lying between $0.5 \mathrm{fm}$ and $1.2 \mathrm{fm}$. This region just interpolates between the short distance domain and normal hadron diameters, thus allowing a natural extension of the phenomenology of hadron-hadron scattering where the model has been applied originally. Model dependent features of the light cone wave functions enter into the magnitude of the production cross section. A good experimental separation of $\sigma_{L}$ and $\sigma_{T}$ can help to obtain a real breakthrough in our understanding of diffractive electroproduction, since the physics of the $q \bar{q}$ pair state is so much different for both photon polarizations. The extension to photoproduction and low $Q^{2}$ electroproduction necessitates a modification of the simple perturbative $q \bar{q}$ wavefunction in the photon.

The model of the stochastic vacuum cannot predict the behaviour of the cross sections as a function of the cm energy $\sqrt{s}$. If all parameters are fixed, it yields constant cross sections. We therefore have confined ourselves in this paper to the energy range $10 \mathrm{GeV} \leq$ $\sqrt{s} \leq 20 \mathrm{GeV}$. The absolute values for the cross sections are correctly reproduced for $\rho$, $\omega$ and $J / \psi$-production. Our results for $\phi$-production are about a factor 2 larger than the NMC data, we can, however, explain the $Q^{2}$-dependence of the ratio of $\phi$ to $\rho$ production as observed by ZEUS 25].

In the present model the energy dependence of the total hadronic cross section and the slope of the elastic cross section can be obtained consistently by increasing the hadron radii (slightly) with energy. Due to the expected difference of the energy dependence of the perturbative and nonperturbative contributions (hard and soft pomeron) one has also to take into account perturbative contributions if one wants to obtain a realistic model of the energy dependence of diffractive electroproduction. This will be done in a forthcoming work.

\section{ACKNOWLEDGMENTS}

We are indebted to Andrzej Sandacz for providing us the NMC data and making clear related issues. We have benefited from discussions with Michael Rueter.

\section{APPENDIX A: PHOTON WAVE FUNCTION COMPUTATION}

The photon wave function in the framework of light cone perturbation theory is discussed in [18. We compute it using the rules and conventions given in [22. For a photon with momentum $q=\left[q^{+}, q^{-}=-Q^{2} / 2 q^{+}, \mathbf{q}=\mathbf{0}\right]$, one multiplies

- a color factor $\sqrt{N_{c}}$,

- a flavor part $e_{f} \delta_{f \bar{f}}$,

- a spinor term $\bar{u}\left(z q^{+}, \mathbf{k}, h\right) \varepsilon_{\mu}(q, \lambda) \gamma^{\mu} v\left((1-z) q^{+},-\mathbf{k}, \bar{h}\right)$, 
- a factor $\left(\sqrt{2} z q^{+}\right)^{-1 / 2}\left(\sqrt{2}(1-z) q^{+}\right)^{-1 / 2}$ for the quark and antiquark lines,

- a light cone energy denominator $-\sqrt{2} q^{+}\left[Q^{2}+\frac{\mathbf{k}^{2}+m^{2}}{z(1-z)}\right]^{-1}$.

The polarization vectors of the photon are $\varepsilon(q, 0)=\left[q^{+} / Q, Q / 2 q^{+}, \mathbf{0}\right]$ and $\varepsilon(q, \pm 1)=$ $[0,0,-1 / \sqrt{2}, \mp i / \sqrt{2}]$. The spinor matrix element between infinite-momentum-frame helicity eigenspinors 22] are

$$
\begin{aligned}
& \bar{u} \gamma^{+} v=2 \sqrt{z(1-z)} q^{+} \delta_{h,-\bar{h}}, \\
& \bar{u} \gamma^{-} v=-\frac{\mathbf{k}^{2}+m^{2}}{\sqrt{z(1-z)} q^{+}} \delta_{h,-\bar{h}}, \\
& \bar{u} \gamma^{i} v=\frac{(1-2 z) k^{i} \mp i \epsilon^{i j 3} k^{j}}{\sqrt{z(1-z)}} \delta_{h,-\bar{h}} \mp m \frac{\delta^{i 1} \mp i \delta^{i 2}}{\sqrt{z(1-z)}} \delta_{h, \bar{h}} .
\end{aligned}
$$

In the last line $i=1,2$ and $\mp$ stands for a minus sign if $h=+1 / 2$ and for a plus sign when $h=-1 / 2$.

By taking the Fourier transform

$$
\psi(z, \mathbf{r})=\int \frac{d^{2} \mathbf{k}}{(2 \pi)^{2}} e^{i \mathbf{k} \cdot \mathbf{r}} \psi(z, \mathbf{k}),
$$

one gets the expressions given in Eq. (3.2). In the longitudinal case there is an additional $\delta^{(2)}(\mathbf{r})$ which one can drop because the color interaction vanishes at 0 transverse distance.

It is of course possible to obtain a wave function description in covariant approach and we want here to give the steps necessary to get the photon wave function. First, it is important to notice that a photon-quark-antiquark coupling in a Feynman graph can be interpreted in term of a photon wave function in light cone perturbation theory if the $x^{+}$-ordering is $\gamma \rightarrow q \bar{q}$. This is the case in the formal limit $q^{+} \rightarrow+\infty$ where this ordering survives.

The first step is the evaluation of the $k^{-}$integral which leads for asymptotic $q^{+}$to

$$
\frac{d k^{+} d^{2} \mathbf{k}}{(2 \pi)^{4}} \int_{-\infty}^{+\infty} d k^{-} \frac{f\left(k^{+}, k^{-}, \mathbf{k}\right)}{\left(k^{2}-m^{2}+i \varepsilon\right)\left((k-q)^{2}-m^{2}+i \varepsilon\right)} \sim \frac{d z d^{2} \mathbf{k}}{16 \pi^{3}} \frac{i f\left(k^{+}, 0^{-}, \mathbf{k}\right)}{\mathbf{k}^{2}+m^{2}-z(1-z) q^{2}}
$$

provided $f\left(k^{-}=0\right)$ is finite and non-zero. The numerator, $N=i(\not k+m)\left(-i e \ddagger_{\lambda}\right) i(\not k-\not k+m)$, is

$$
\begin{aligned}
N & =i e\left\{k \cdot \varepsilon(2 \not k-\not 1)-k \cdot(k-q) \notin+i \epsilon_{\alpha \mu \nu \rho} \gamma_{5} \gamma^{\alpha} k^{\mu} \varepsilon^{\nu} q^{\rho}+m(2 k \cdot \varepsilon-\notin \not 1+m \notin)\right\} \\
& \approx i e q^{+} \gamma^{-}\left\{\frac{\delta_{\lambda 0}}{Q}\left[z(1-z) q^{2}+\mathbf{k}^{2}+m^{2}\right]+(1-2 z) \mathbf{k} \cdot \varepsilon_{T}+i \gamma_{5} \epsilon^{i j 3} k^{i} \varepsilon_{T}^{j}+m \ddagger_{T}\right\} .
\end{aligned}
$$

The wave function is obtained by taking the helicity matrix element $\bar{w}(h) N w(-\bar{h}) / \sqrt{2} q^{+}$ with $w(1 / 2)=(1 / \sqrt{2}, 0,-1 / \sqrt{2}, 0)$ and $w(-1 / 2)=(0,1 / \sqrt{2}, 0,1 / \sqrt{2})$ 22. 


\section{APPENDIX B: HADRON WAVE FUNCTION PARAMETERS}

The value of the wave function at the origin is related to the meson leptonic decay constant

$$
\left\langle 0\left|J^{\mu}(0)\right| V(q, \lambda)\right\rangle=e f_{V} M_{V} \varepsilon^{\mu}(q, \lambda),
$$

which appears in the expression of the vector meson $e^{+} e^{-}$width

$$
\Gamma\left(V \rightarrow e^{+} e^{-}\right)=\frac{4 \pi \alpha^{2}}{3 M_{V}} f_{V}^{2} .
$$

In the parametrization Eq. (3.3), this constraint leads to the determination of the parameter $\mathcal{N}$. The fixing condition, which depends on the meson helicity, $\lambda$, is

$$
1=\int_{0}^{1} d z z(1-z) f_{L}(z)=\int_{0}^{1} d z \frac{2\left[z^{2}+(1-z)^{2}\right] \omega_{T}^{2}+m^{2}}{2 M_{V}^{2} z(1-z)} f_{T}(z),
$$

with $\hat{e}_{V}$ the effective quark charge in the meson $V$ expressed in units of the proton charge (see Table 【).

The normalization condition is

$$
\left\langle V\left(q^{\prime}, \lambda^{\prime}\right) \mid V(q, \lambda)\right\rangle=(2 \pi)^{3} 2 q^{+} \delta\left(q^{+}-q^{++}\right) \delta^{(2)}\left(\mathbf{q}-\mathbf{q}^{\prime}\right) \delta_{\lambda \lambda^{\prime}},
$$

which leads to the relation

$$
\omega_{\lambda}=\frac{\pi f_{V}}{\sqrt{2 N_{c}} \hat{e}_{V}} \sqrt{I_{\lambda}}
$$

where

$$
\begin{aligned}
& I_{L}=\int_{0}^{1} d z z^{2}(1-z)^{2} f_{L}^{2}(z), \\
& I_{T}=\int_{0}^{1} d z \frac{\left[z^{2}+(1-z)^{2}\right] \omega_{T}^{2}+m^{2}}{M_{V}^{2}} f_{T}^{2}(z) .
\end{aligned}
$$

$\omega_{\lambda}$ and $\mathcal{N}_{\lambda}$ are therefore defined by a system of implicite equations, Eq. (B2) and Eq. (B4). Solutions are listed in Table [ 1 together with the corresponding root mean square radius $R=\left\langle R_{3}^{2}\right\rangle^{1 / 2}=\sqrt{3 / 2} / 2 \omega$. 


\begin{tabular}{lccccccc}
\hline \hline $\begin{array}{l}V\left(M_{V}\right) \\
(\mathrm{GeV})\end{array}$ & $\hat{e}_{V}$ & $\begin{array}{c}\Gamma \\
\mathrm{keV}\end{array}$ & $\begin{array}{c}f_{V} \\
\mathrm{MeV}\end{array}$ & $\begin{array}{c}\omega_{L} \\
\mathrm{GeV}\end{array}$ & $\begin{array}{c}R_{L} \\
\mathrm{fm}\end{array}$ & $\begin{array}{c}\omega_{T} \\
\mathrm{GeV}\end{array}$ & $\begin{array}{c}R_{T} \\
\mathrm{fm}\end{array}$ \\
\hline$\rho(770)$ & $1 / \sqrt{2}$ & $6.7(7)$ & 153 & .33 & .37 & .22 & .55 \\
$\omega(782)$ & $1 / 3 \sqrt{2}$ & 0.60 & 45.8 & .30 & .40 & .21 & .58 \\
$\phi(1019)$ & $1 / 3$ & 1.37 & 79.1 & .37 & .33 & .26 & .46 \\
$J / \psi(3097)$ & $2 / 3$ & $5.2(6)$ & 270 & .68 & .18 & .57 & .21 \\
\hline \hline
\end{tabular}

TABLE I. Vector meson characteristics. The quark masses considered are $m_{u}=m_{d}=0$, $m_{s}=0.15 \mathrm{GeV}, m_{c}=1.3 \mathrm{GeV}$.

\section{REFERENCES}

[1] H.G. Dosch, Phys. Lett. B 190, 177 (1987); H.G. Dosch and Y.A. Simonov, ibid. 205, 339 (1988).

[2] H.G. Dosch, E. Ferreira and A. Krämer, Phys. Rev. D 50, 1992 (1994).

[3] O. Nachtmann, Ann. Phys. 209, 436 (1991).

[4] M. Rueter and H.G. Dosch, Z. Phys. C 66, 245 (1995).

[5] B. Povh and J. Hüfner, Phys. Rev. Lett. 58, 1612 (1987).

[6] Work in progress.

[7] A. Donnachie and P.V. Landshoff, Phys. Lett. B 185, 403 (1987).

[8] A. Donnachie and P.V. Landshoff, Phys. Lett. B 348, 213 (1995).

[9] B.Z. Kopeliovich et al. Phys. Lett. B 309, 179 (1993); J. Nemchick, N.N. Nikolaev and B.G. Zakharov, Phys. Lett. B 341, 228 (1994).

[10] M.G. Ryskin, Z. Phys. C 57, 89 (1993).

[11] S.J. Brodsky et al., Phys. Rev. D 50, (1994).

[12] A.H. Mueller, Nucl. Phys. B415, 373 (1994); N.N. Nikolaev and B.G. Zakharov, Z. Phys. C 64, 631 (1994).

[13] H.G. Dosch, Progr. in Part. a. Nucl. Phys. 33, 121 (1994).

[14] N.G. Van Kampen, Phys. Rep. 24, 172 (1976).

[15] A. Di Giacomo and H. Panagopoulos, Phys. Lett. B 285, 133 (1992).

[16] J.F. Gunion and D.E. Soper, Phys. Rev. D 15, 2617 (1977).

[17] M. Rueter and H.G. Dosch, Phys. Lett. B 380, 177 (1996).

[18] J.D. Bjorken, J.B. Kogut and D.E. Soper, Phys. Rev. D 3, 1382 (1971).

[19] G.P. Lepage et al., in Particles and Fields 2, Proceedings of the Banff Summer Institute, Banff, Canada, 1981, edited by A.Z. Capri and A.N. Kamal (Plenum,New York,1983).

[20] A. Di Giacomo, E. Meggiolaro, H. Panagopoulos, Field strength correlations in the QCD vacuum at short distances, hep-lat 9603017.

[21] N.N. Nikolaev and B.G. Zakharov, Z. Phys. C 49, 607 (1991).

[22] G.P. Lepage and S.J. Brodsky, Phys. Rev. D 22, 2157 (1980).

[23] M. Wirbel, B. Stech and M. Bauer, Z. Phys. C 29, 637 (1985).

[24] NMC, M. Arneodo et al., Nucl. Phys. B429, 503 (1994).

[25] ZEUS Collab., M. Derrick et al, Phys. Lett. B 380, 220 (1996).

[26] EMC, J.J. Aubert et al., Nucl. Phys. B213, 1 (1983).

[27] M. Binkley et al., Phys. Rev. Lett. 48, 73 (1982).

[28] NMC, M. Arneodo et al., Phys. Lett. B 332, 195 (1994). 